\title{
A HIGH-RESOLUTION X-RAY AND OPTICAL STUDY OF SN 1006: ASYMMETRIC EXPANSION AND SMALL-SCALE STRUCTURE IN A TYPE IA SUPERNOVA REMNANT**
}

\author{
P. Frank Winkler ${ }^{1}$, Brian J. Williams ${ }^{2}$, Stephen P. Reynolds ${ }^{3}$, Robert Petre ${ }^{2}$, \\ KnOx S. Long ${ }^{4}$, SAtoru Katsuda ${ }^{5}$, And Una Hwang ${ }^{2}$ \\ ${ }^{1}$ Department of Physics, Middlebury College, Middlebury, VT 05753, USA; winkler@middlebury.edu \\ ${ }^{2}$ NASA Goddard Space Flight Center, Greenbelt, MD 20771, USA; brian.j.williams@ nasa.gov, robert.petre-1@ nasa.gov \\ ${ }_{3}^{3}$ Physics Department, North Carolina State University, Raleigh, NC 27695, USA; reynolds@ncsu.edu \\ ${ }^{4}$ Space Telescope Science Institute, 3700 San Martin Drive, Baltimore, MD 21218, USA; long@ stsci.edu \\ ${ }^{5}$ RIKEN (The Institute of Physical and Chemical Research), 2-1 Hirosawa, Wako, Saitama 351-0198, Japan \\ Received 2013 October 15; accepted 2013 December 10; published 2014 January 9
}

\begin{abstract}
We introduce a deep (670 ks) X-ray survey of the entire SN 1006 remnant from the Chandra X-Ray Observatory, together with a deep $\mathrm{H} \alpha$ image of SN 1006 from the $4 \mathrm{~m}$ Blanco telescope at CTIO. Comparison with Chandra images from 2003 gives the first measurement of the X-ray proper motions around the entire periphery, carried out over a $9 \mathrm{yr}$ baseline. We find that the expansion velocity varies significantly with azimuth. The highest velocity of $\sim 7400 \mathrm{~km} \mathrm{~s}^{-1}$ (almost 2.5 times that in the northwest (NW)) is found along the southeast (SE) periphery, where both the kinematics and the spectra indicate that most of the X-ray emission stems from ejecta that have been decelerated little, if at all. Asymmetries in the distribution of ejecta are seen on a variety of spatial scales. Si-rich ejecta are especially prominent in the SE quadrant, while $\mathrm{O}$ and $\mathrm{Mg}$ are more uniformly distributed, indicating large-scale asymmetries arising from the explosion itself. Neon emission is strongest in a sharp filament just behind the primary shock along the NW rim, where the pre-shock density is highest. Here the Ne is likely interstellar, while $\mathrm{Ne}$ within the shell may include a contribution from ejecta. Within the interior of the projected shell we find a few isolated "bullets" of what appear to be supernova ejecta that are immediately preceded by bowshocks seen in $\mathrm{H} \alpha$, features that we interpret as ejecta knots that have reached relatively dense regions of the surrounding interstellar medium, but that appear in the interior in projection. Recent three-dimensional hydrodynamic models for Type Ia supernovae display small-scale features that strongly resemble the ones seen in X-rays in SN 1006; an origin in the explosion itself or from subsequent hydrodynamic instabilities both remain viable options. We have expanded the search for precursor X-ray emission ahead of a synchrotron-dominated shock front, as expected from diffusive shock acceleration theory, to numerous regions along both the northeast and southwest rims of the shell. Our data require that a precursor be thinner than about $3^{\prime \prime}$, and fainter than about $5 \%$ of the post-shock peak. These limits suggest that the magnetic field is amplified by a factor of seven or more in a narrow precursor region, promoting diffusive particle acceleration.
\end{abstract}

Key words: ISM: individual objects (SN 1006) - ISM: kinematics and dynamics - ISM: supernova remnants supernovae: individual (SN 1006) - X-rays: individual (SN1006) - X-rays: ISM

Online-only material: animation, color figures

\section{INTRODUCTION}

X-ray investigation of the SN 1006 remnant began with the suggestion that a bump on one edge of the extended Lupus Loop supernova remnant (SNR), observed during a 1971 rocket flight by the Livermore group, might be attributed to SN 1006 (Palmieri et al. 1972). Positive identification followed from the X-ray experiment on the $O S O-7$ satellite (Winkler \& Laird 1976), and it was included in the final Uhuru catalog (Forman et al. 1978). Its flux, about $3 \%$ of that from Cas A and $\lesssim 0.2 \%$ of that from the Crab Nebula, made it among the faintest sources identified by the first generation of X-ray astronomy satellites.

After that humble beginning, SN 1006 was studied with virtually all major X-ray satellites through the next two decades: SAS-3 (Winkler et al. 1979), the Einstein Observatory (Pye et al. 1981), ROSAT (Willingale et al. 1996; Winkler \& Long 1997), and others. The seeming discord between a remnant with a clear bilateral shell in radio maps, yet with a featureless power-law

\footnotetext{
* Based on observations made with NASA's Chandra X-Ray Observatory, operated by the Smithsonian Astrophysical Observatory under contract \# NAS83060; the data were obtained through program GO1-12115.
}

spectrum in its bright X-ray regions, led to a well-known paper from the Einstein/OSO-8 era, "Is the remnant of SN 1006 Crablike?" (Becker et al. 1980). The definitive answer came from Koyama et al. (1995), whose spatially resolved spectroscopy using ASCA showed that the X-ray-bright northeast (NE) and southwest (SW) shell limbs are indeed power-law dominated, but that emission from the interior and the remaining shell is dramatically different: soft, thermal X-rays more typical of SNRs. This finding provided the first clear evidence for diffusive shock acceleration of electrons to high energies in SNR shocks, and cemented the long-suspected link between supernovae (SN) and cosmic rays.

SN 1006 is relatively close at $2.2 \mathrm{kpc}$ (Winkler et al. 2003), where the spatial scale is $1^{\prime \prime} \approx 0.01 \mathrm{pc}$, and since it is located 14.6 above the Galactic plane it has relatively low foreground absorption, $N_{\mathrm{H}} \approx 7 \times 10^{20} \mathrm{~cm}^{-2}$ (Dubner et al. 2002; Uchida et al. 2013). ${ }^{6}$ Its location, low-density surroundings far from any

\footnotetext{
6 Nikolić et al. (2013) have recently argued for a shorter distance of $1.7 \mathrm{kpc}$ to SN 1006, based on new optical spectra and model calculations from van Adelsberg et al. (2008). We use the $2.2 \mathrm{kpc}$ distance throughout this paper; scaling to a shorter distance is straightforward.
} 
recent star formation, apparent absence of any compact remnant, and the implication from Chinese records that it remained visible for several years (Stephenson \& Clark 2002) all indicate that it was a Type Ia event; it is the closest remnant of a historical SN Ia.

All these attributes have made it an important target for continuing investigation from the current generation of X-ray telescopes: Chandra, XMM-Newton, and Suzaku. The first Chandra observations were a pair of deep pointings in 2000 and 2001 with the Advanced CCD Imaging Spectrometer (ACIS) S-array along the contrasting nonthermal NE and thermal northwest (NW) rims in 2001, reported by Long et al. (2003), followed in 2003 by a mosaic of ACIS-I fields that covered the entire remnant (Cassam-Chenaï et al. 2008), and in 2008 by a second-epoch ACIS-S observation of the NE rim (Katsuda et al. 2009). The Chandra observations we report here were designed as a follow-up to those by Cassam-Chenaï et al. (2008) with nearly five times longer exposure around most of the rim, in order to provide both a more detailed look at small-scale features and a second epoch for measuring the expansion.

From XMM-Newton, a very deep set of imaging observations has been presented by Miceli et al. (2012, 2013a) and references therein, while observations with the reflection grating spectrometer have focused on a prominent knot of ejecta along the NW shock front (Vink et al. 2003; Broersen et al. 2013). Finally, Suzaku results focusing on spatially resolved spectroscopy have been reported by Yamaguchi et al. (2008), Bamba et al. (2008), and Uchida et al. (2013). Results from these missions that are most relevant to the present paper will be mentioned in the context of the new Chandra data in subsequent sections.

In this paper we present the first results from the complete Chandra Cycle 13 Large Project Observation of SN 1006, comprising pointings at 10 overlapping fields with the ACIS for a total of $670 \mathrm{ks}$. We also present the images resulting from a deep optical study from the $4 \mathrm{~m}$ Blanco telescope and Mosaic II camera at CTIO, carried out in 2010 April. Conceived together, these are intended to give a detailed high-resolution view of this important remnant in multiple bands, to which we plan to add high-resolution radio images to be carried out from the Jansky Very Large Array in its three hybrid configurations over the next two years. In Sections 2 and 3 of this paper we briefly describe the X-ray and optical observations and data reduction, and present the complete mosaic images.

We then highlight some results that are immediately apparent, and suggest areas for future work by ourselves and others. In Section 4 we discuss some of the X-ray and optical features that the new images reveal, including what appear to be "bullets" of ejecta preceded by small optical bowshocks. Section 5 presents the first X-ray expansion measurement around the entire rim of the $30^{\prime}$ diameter shell; Section 6 presents narrowband X-ray images in lines corresponding to different important elements, with inferences for the distribution of ejecta; and Section 7 presents limits on precursor X-ray emission ahead of the synchrotron-dominated shocks. We present a discussion of these results and implications for SN 1006 in particular, and for Type Ia $\mathrm{SNe}$ in general, in Section 8. Finally, Section 9 summarizes our conclusions from this paper.

\section{X-RAY OBSERVATIONS FROM THE CHANDRA/ACIS}

The goal of the new Chandra observations was to provide a detailed picture of the fine-scale structure of the X-ray remnant and to provide a second-epoch image in order to measure the expansion around the entire shell. A series of deep ACIS
Table 1

Chandra/ACIS Observations of SN 1006

\begin{tabular}{lcccrcr}
\hline \hline ObsID $^{\text {a }}$ & Array & $\begin{array}{c}\text { R.A. } \\
(\text { J2000. })\end{array}$ & $\begin{array}{c}\text { Decl. } \\
(\mathrm{J} 2000 .)\end{array}$ & Roll & Obs. Date & $\begin{array}{c}\text { Exposure } \\
(\mathrm{ks})\end{array}$ \\
\hline 9107 & ACIS-S & $15: 03: 51.5$ & $-41: 51: 19$ & 280.4 & 2008 Jun 24 & 89.0 \\
13737 & ACIS-S & $15: 02: 15.9$ & $-41: 46: 10$ & $31: 7$ & 2012 Apr 20 & 87.1 \\
13738 & ACIS-I & $15: 01: 41.8$ & $-41: 58: 15$ & $25: 3$ & 2012 Apr 23 & 73.5 \\
14424 & ACIS-I & $15: 01: 41.8$ & $-41: 58: 15$ & $25: 3$ & 2012 Apr 27 & 25.4 \\
13739 & ACIS-I & $15: 02: 12.6$ & $-42: 07: 01$ & 9.1 & 2012 May 04 & 100.1 \\
13740 & ACIS-I & $15: 02: 40.7$ & $-41: 50: 21$ & 294.5 & 2012 Jun 10 & 50.4 \\
13741 & ACIS-I & $15: 03: 48.0$ & $-42: 02: 53$ & 24.6 & 2012 Apr 25 & 98.5 \\
13742 & ACIS-I & $15: 03: 01.8$ & $-42: 08: 27$ & 289.1 & 2012 Jun 15 & 79.0 \\
13743 & ACIS-I & $15: 03: 01.8$ & $-41: 43: 05$ & 19.9 & 2012 Apr 28 & 92.6 \\
14423 & ACIS-I & $15: 02: 50.9$ & $-41: 55: 25$ & $21: 2$ & 2012 Apr 25 & 25.0 \\
14435 & ACIS-I & $15: 03: 42.5$ & $-41: 54: 49$ & $297: 3$ & 2012 Jun 08 & 38.3
\end{tabular}

Notes. ${ }^{\text {a }}$ For ObsID 9107, Petre was PI; for the others, PI was Winkler.

pointings was planned to cover the entire $30^{\prime}$ diameter remnant, with most of the aim points located just inside the shell rim, spaced to ensure coverage of the entire rim close enough to onaxis to achieve a resolution $\lesssim 5^{\prime \prime}$. The S-array was used for the NE and NW rims (ObsIDs 9107 in 2009 and ObsID 13737 in 2012, respectively) in order to match earlier ACIS-S pointings for proper-motion measurements along those parts of the shell (Long et al. 2003). Results of both these measurements have already been reported by Katsuda et al. (2009, 2010, 2013).

All the other pointings were made with the ACIS-I array and were carried out in 2012 April-July. The strategy was similar to that used by Cassam-Chenaï et al. (2008) for their ACIS-I mosaic, except that most of our pointings were 80-100 ks instead of $20 \mathrm{ks}$, and ours emphasized positions near the shell rim in order to achieve optimum angular resolution there. We also benefited from their earlier image in being able to position the detector to avoid having critical features fall onto chip gaps in the ACIS detector. Despite the deep second-epoch observation of the NE rim in 2008, we included short observations of this bright region in 2012 in order to ensure contemporary observations of the entire shell. A complete journal of the new observations is provided in Table 1, and a map showing the relative exposure is in Figure 1. All the data were processed through the standard Chandra pipeline, and then reprocessed using CIAO version 4.5 and CALDB 4.5.6, in order to assure that the latest gain and quantum-efficiency corrections are used.

\subsection{Aspect Correction and X-Ray Mosaics}

In order to correct the data to a uniform, absolute coordinate system that can be used for precise comparison with optical and other data, we compiled a list of astrometric optical sources from the NOMAD catalog (Zacharias et al. 2005), selected for

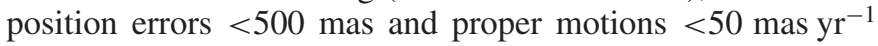
in both R.A. and decl. For each ObsID we used the CIAO task wavdetect to locate point sources in the field, and from these selected only ones with significance $>5 \sigma$. We then used the CIAO task wcs_match to match the source list for each field against our selected list from the NOMAD catalog, and to calculate translations to achieve the optimum match. ${ }^{7}$ For most of the X-ray pointings there were four to six excellent source matches that yielded aspect translations of $<1.4$ ACIS pixels $\left(<0^{\prime \prime} \cdot 7\right)$ in both coordinate directions. For three pointings,

\footnotetext{
7 The wcs_match task has an option to calculate transformations that include rotations and scale changes, but we used the translation-only method, which is more stable for a small number of sources.
} 


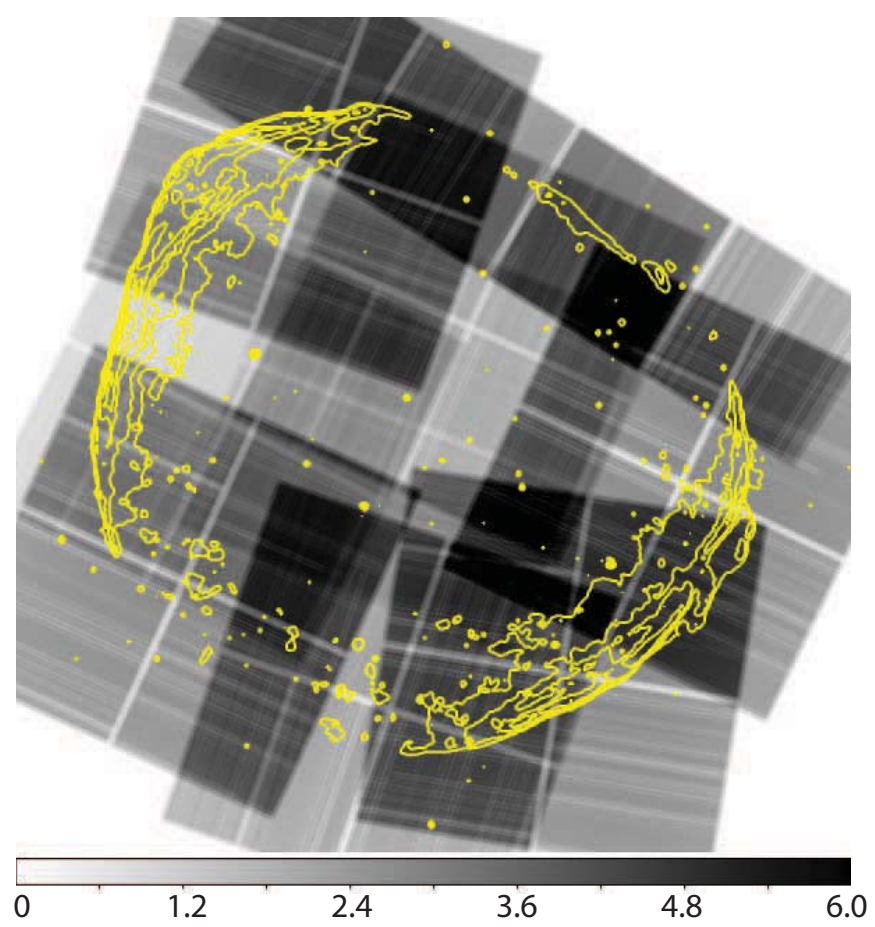

Figure 1. Exposure map for the 2012 ACIS observations. Contours representing the broad X-ray flux are overlaid. The exposure time in most of the individual pointings ranges from $80 \mathrm{ks}$ to $100 \mathrm{ks}$, but the exposure is, of course, deeper where multiple pointings overlap. The scale gives the exposure in units $10^{7} \mathrm{~cm}^{2} \mathrm{~s}$.

(A color version of this figure is available in the online journal.)

however (ObsIDs 13738, 13743. and 14423), there were not enough reliable source matches, so no aspect correction was applied. Comparison of the wavdetect positions for point $\mathrm{X}$-ray sources detected with high significance in overlapping ObsIDs (not limited to sources with optical counterparts) showed agreement to within $<1$ ACIS pixel $\approx 0$ "'.5 rms.

We then combined all the aspect-corrected and reprocessed 2012 data into mosaic images of SN 1006 in several energy bands using the CIAO script merge_obs. The resulting mosaic image in the soft $(0.5-1.2 \mathrm{keV}$, shown in red), medium (1.2-2.0 keV, shown in green), and hard (2.0-7.0 keV, shown in blue) bands is shown in Figure 2.

\section{OPTICAL IMAGES FROM CTIO}

The most prominent optical emission from SN 1006 is a relatively bright, delicate filament extending along much of the NW limb of the shell (van den Bergh 1976; Long et al. 1988; Raymond et al. 2007) and seen only in the Balmer lines of hydrogen (Schweizer \& Lasker 1978; Ghavamian et al. 2002). In addition, Winkler et al. (2003) reported far fainter and less distinct Balmer emission surrounding most of the shell, and there is an even fainter diffuse oval of emission, probably associated with SN 1006, filling much of the northern half of the remnant. The "nonradiative" emission like that from SN 1006 results from at least partially neutral pre-shock $\mathrm{H}$ atoms that traverse the shock, where they can undergo either direct collisional excitation, or charge exchange with hot post-shock protons-processes that produce narrow and broad emissionline components, respectively (Chevalier \& Raymond 1978; Heng 2010 for a recent review).

In order to study the emission from the entire remnant in greater detail, we carried out deep optical imaging of SN 1006 in
Table 2

CTIO Observations, 4 m Blanco Telescope ${ }^{\mathrm{a}}$

\begin{tabular}{|c|c|c|c|c|}
\hline \multicolumn{4}{|c|}{ Filter } & \multirow[b]{2}{*}{$\begin{array}{c}\text { Exposure } \\
\text { (s) }\end{array}$} \\
\hline $\begin{array}{l}\text { Informal } \\
\text { Name }\end{array}$ & $\begin{array}{c}\text { CTIO } \\
\text { Designation }\end{array}$ & $\begin{array}{l}\lambda_{c}^{\mathrm{b}} \\
(\AA)\end{array}$ & $\begin{array}{l}\Delta \lambda^{\mathrm{b}} \\
(\AA)\end{array}$ & \\
\hline $\mathrm{H} \alpha$ & c6009 & 6563 & 80 & $24 \times 600$ \\
\hline $\mathrm{H} \alpha+8 \mathrm{~nm}$ & c6011 & 6650 & 80 & $22 \times 600$ \\
\hline
\end{tabular}

Notes.

a 2010 April 15-18; observers: Winkler \& Long.

${ }^{\mathrm{b}}$ Central wavelength and FWHM in the $f / 2.8$ telescope beam.

two narrow bands: $\mathrm{H} \alpha$ and a matched continuum for subtracting the stars to reveal the faintest Balmer emission features. The observations, in 2010 April, used the $4 \mathrm{~m}$ Blanco telescope at CTIO and Mosaic II camera, whose field of $37^{\prime} \times 37^{\prime}$, at a scale of 0.27 pixel $^{-1}$, is well matched to the size of the remnant. The filters were centered at $6563 \AA$ and $6650 \AA$, respectively, both with a bandwidth of $80 \AA$ (FWHM). We obtained 24 10-minute exposures in $\mathrm{H} \alpha$, and 22 10-minute ones in the continuum, dithered by a few arcmin between exposures to cover a somewhat larger total field and to improve the flat fielding. The observational details are summarized in Table 2 .

The images were processed through the standard NOAO Mosaic pipeline; subsequently we determined a more precise World Coordinate System using stars from the UCAC4 catalog (Zacharias et al. 2013), selected for position errors $<100$ mas

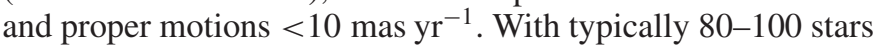
on each of the eight chips in each Mosaic frame, we obtained excellent fits, with rms uncertainty that was typically $\lesssim 60$ mas in both R.A. and decl. Both the $\mathrm{H} \alpha$ and continuum frames were then reprojected onto a standard system at a scale of $0^{\prime \prime} .2$ pixel $^{-1}$, and stacked to produce mosaic images, using IRAF $^{8}$ tasks in the mscred package. Finally, we scaled and subtracted the continuum image from the $\mathrm{H} \alpha$ one, to give the image shown in Figure 3.

The basic morphology of optical emission from SN 1006 is, naturally, entirely consistent with previous observations. The brightest segments of the NW filament have $\mathrm{H} \alpha$ surface brightness 3.5-4 $\times 10^{-16} \mathrm{erg} \mathrm{cm}^{-2} \mathrm{~s}^{-1} \operatorname{arcsec}^{-2}$. In our earlier deep image, taken in 1998 from the 0.6/0.9 m Curtis Schmidt telescope at CTIO, we measured about half this surface brightness (Winkler et al. 2003); since the sharpest and brightest segments were blurred in the lower-resolution Schmidt images, the values from the two observations are entirely consistent. The far fainter and more diffuse parts of the rim that are clearly visible in the south and elsewhere have surface brightness $\sim 1 \times 10^{-17} \mathrm{erg} \mathrm{cm}^{-2} \mathrm{~s}^{-1} \operatorname{arcsec}^{-2}$, fainter by a factor of $\sim 40$ than the brightest ones. Our 1998 Schmidt image shows these features as well, at about the same surface brightness but with lower signal-to-noise (Figure 5 of Winkler et al. 2003). For reference, we show both the 1998 and 2010 continuumsubtracted images at the same scale in the lower two panels of Figure 3. The images from both epochs show additional faint structures; e.g., the previously mentioned faint, diffuse oval of emission that fills much of the northern half of the shell, and an irregular band of emission that wraps around the southern half of the shell, about $5^{\prime}$ inside the rim. The latter connects to emission that extends far beyond the shell to the west and north, and that

\footnotetext{
8 IRAF is distributed by the National Optical Astronomy Observatory, which is operated by AURA, Inc., under cooperative agreement with the National Science Foundation.
} 


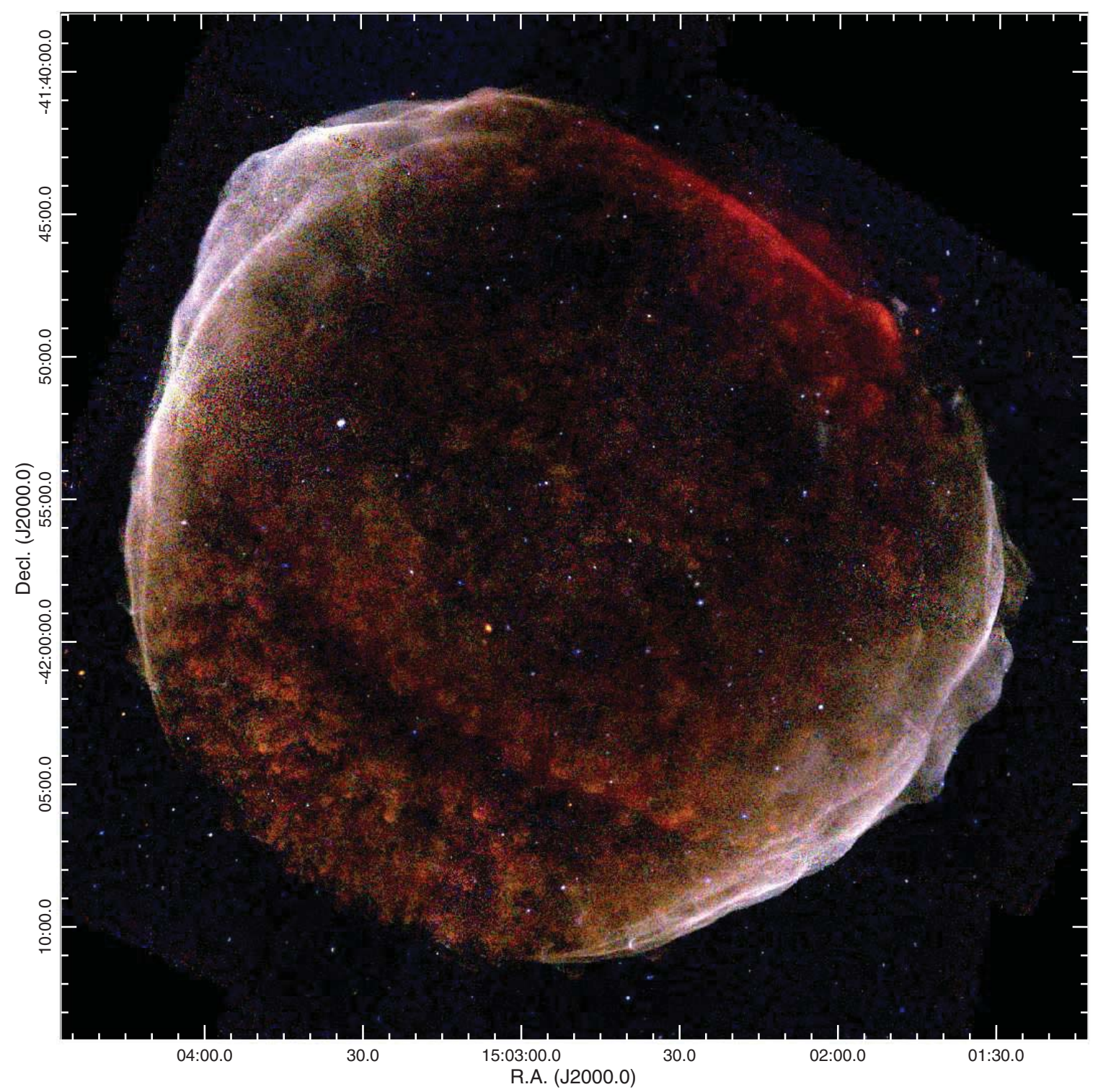

Figure 2. "True-color" X-ray mosaic of all the 2012 Chandra/ACIS observations of SN 1006; red $=$ soft $(0.5-1.2 \mathrm{keV})$, green $=$ medium $(1.2-2.0 \mathrm{keV})$, blue $=$ hard $(2.0-7.0 \mathrm{keV})$. The synchrotron-dominated regions along the NE and SW rims are much harder than the thermal-dominated emission from elsewhere in SN 1006.

is presumably in the foreground or background and not physically associated with SN 1006 itself. It is not clear just which emission features within the shell are physically associated, but some-ones with associated X-ray features-definitely are, as we discuss in the following section.

\section{RELATION OF X-RAY AND OPTICAL FEATURES}

Comparison between the X-ray and optical images shows several thin arcs of Balmer emission, primarily within the southern portion of the SN 1006 shell, that lie immediately in front of some of the brighter tufts or flocculi of X-ray emission. These X-ray structures, also seen in previous X-ray images, have scales that are typically $10^{\prime \prime}-30^{\prime \prime}(0.1-0.3 \mathrm{pc})$. Two examples are shown in Figure 4. These Balmer filaments seen (in projection) in the remnant interior strongly resemble bowshocks, and the X-ray tufts behind them have spectra indicating that they are ejecta-dominated (see Section 6). These are probably similar structures to the far brighter bulge in the NW Balmer filament, at about 2 o'clock in Figure 3, which precedes a bright thermal
X-ray knot that has long been attributed to an ejecta bullet (Long et al. 2003; Vink et al. 2003; Broersen et al. 2013).

The presence of Balmer emission absolutely requires partially neutral interstellar $\mathrm{H}$ ahead of the shock, so the bowshock features must be located on the front or back sides of the remnant's shell, seen in the interior only in projection. The X-ray knots behind them have a somewhat flattened appearance, consistent with ejecta running into interstellar material. There are many small X-ray tufts similar to those shown in Figure 4, and with a spectral character that indicates SN ejecta, but that are not preceded by optical bowshocks. This absence simply indicates the absence of neutral gas in front of them; they may not have reached the remnant shell, or the pre-shock gas at that point could be fully ionized or too tenuous to produce significant Balmer emission. The origin of the X-ray tufts-whether with or without associated Balmer bowshocks-is not obvious; they could have resulted from small-scale density inhomogeneities imprinted during the explosion itself (Orlando et al. 2012), or they could be the result of more recent Rayleigh-Taylor (R-T) instabilities in the expanding ejecta (e.g., Warren \& Blondin 

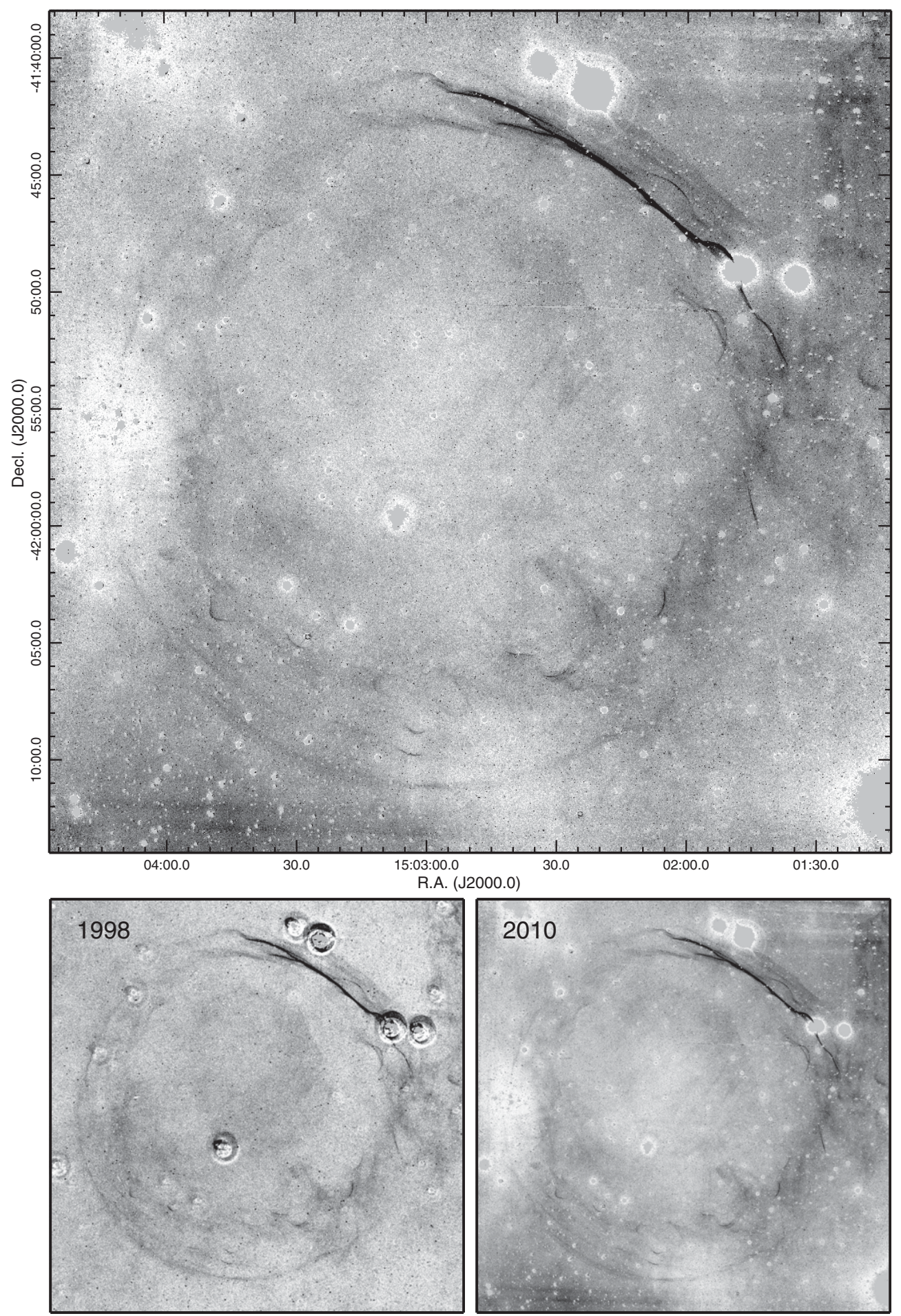

Figure 3. Large upper panel shows a very deep $\mathrm{H} \alpha$ image of SN 1006, after continuum subtraction, obtained at the CTIO 4 m Blanco telescope with the Mosaic II camera, 2010. The relatively bright filaments to the NW are saturated in this display, in order to emphasize the far fainter emission elsewhere in the remnant. The field is $36^{\prime}$ square, and exactly matches that of the X-ray image, Figure 2. The smaller images below show both the 1998 and 2010 images at the same scale; most of the features seen in the 2010 image are also visible in the earlier low-resolution image.

2013). We discuss these possibilities further in Section 8.3. There are also several thin arcs of Balmer emission without an obvious X-ray knot behind, which could have resulted from less dense clumps of ejecta or ones that have dissipated.

In the NW, the new $\mathrm{H} \alpha$ image clearly shows the complex structure ahead of the bright filaments, best shown in Figure 5 (center), where this region is displayed with a very hard stretch to show the faintest emission. Very faint X-ray emission is also seen outside the main Balmer filament, up to the outermost limit of optical emission. The optical morphology indicates a rippled sheet seen edge-on, with the multiple edges representing tangencies at different locations (as shown by Hester 1987). It has long been clear that this is the cause for the undulating structure of the primary NW filament, but the deeper image shows this structure to be more complex than previously realized. The bright filament is the result of an encounter 

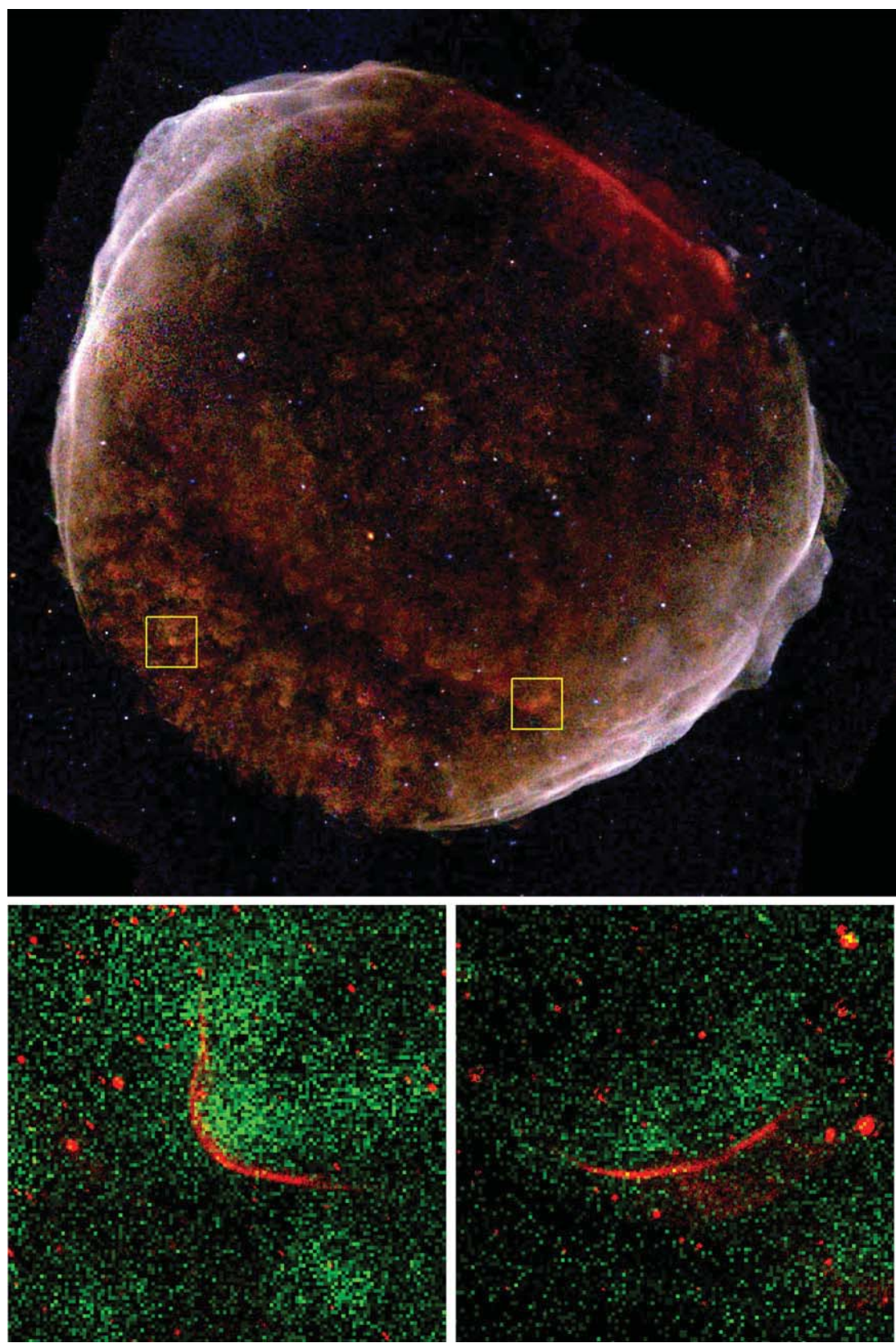

Figure 4. Some of the bright tufts of soft X-ray emission appear immediately behind what appear to be bowshocks seen in H $\alpha$; the two lower panels show two examples, from locations indicated by the yellow boxes in the upper panel. In the lower panels, $\mathrm{H} \alpha$ emission is shown in red, and 0.5-7.0 keV X-rays in green. Both these panels are $2^{\prime}$ square.

between the primary SN shock and a denser ambient medium than around most of SN 1006, and produces the rather flattened structure and slower expansion than elsewhere (Katsuda et al. 2013), as well as the only IR emission seen in SN 1006 (24 $\mu \mathrm{m}$ emission just behind the Balmer filament; Winkler et al. 2013). However, the more complex structure indicates that the dense region that has led to the bright Balmer, X-ray, and IR emission in the NW must not be a "wall," but is instead limited in extent along the line of sight, so that the primary shock has passed well beyond it on either the front or back side of the shell.

\section{X-RAY PROPER-MOTION MEASUREMENTS}

A proper-motion measurement for the bright, synchrotrondominated east-NE (E-NE) rim was reported by Katsuda et al. (2009), and a similar measurement for the thermal-dominated
NW, using an observation from the current project as the second epoch, by Katsuda et al. (2013). Both these measurements compared many individual features in comparably deep ACIS-S observations: epochs 2000-2008 for the E-NE, and 2001-2012 for the NW. The results showed that in the NW, the brightest $\mathrm{X}$-ray filaments that lie just within the bright Balmer filaments have a velocity of $\sim 3000 \mathrm{~km} \mathrm{~s}^{-1}$, essentially the same as measured by Winkler et al. (2003) for the near-coincident Balmer filaments, but that two fainter knots in the NW have nonthermal spectra and much higher velocities: $\sim 5000 \mathrm{~km} \mathrm{~s}^{-1}$, essentially the same as measured for the synchrotron-dominated filaments in the NE. ${ }^{9}$

\footnotetext{
9 As previously mentioned, throughout this paper we assume a distance of $2.2 \mathrm{kpc}$ for converting from proper motion to shock velocity, in part to facilitate comparison with previous proper-motion studies.
} 

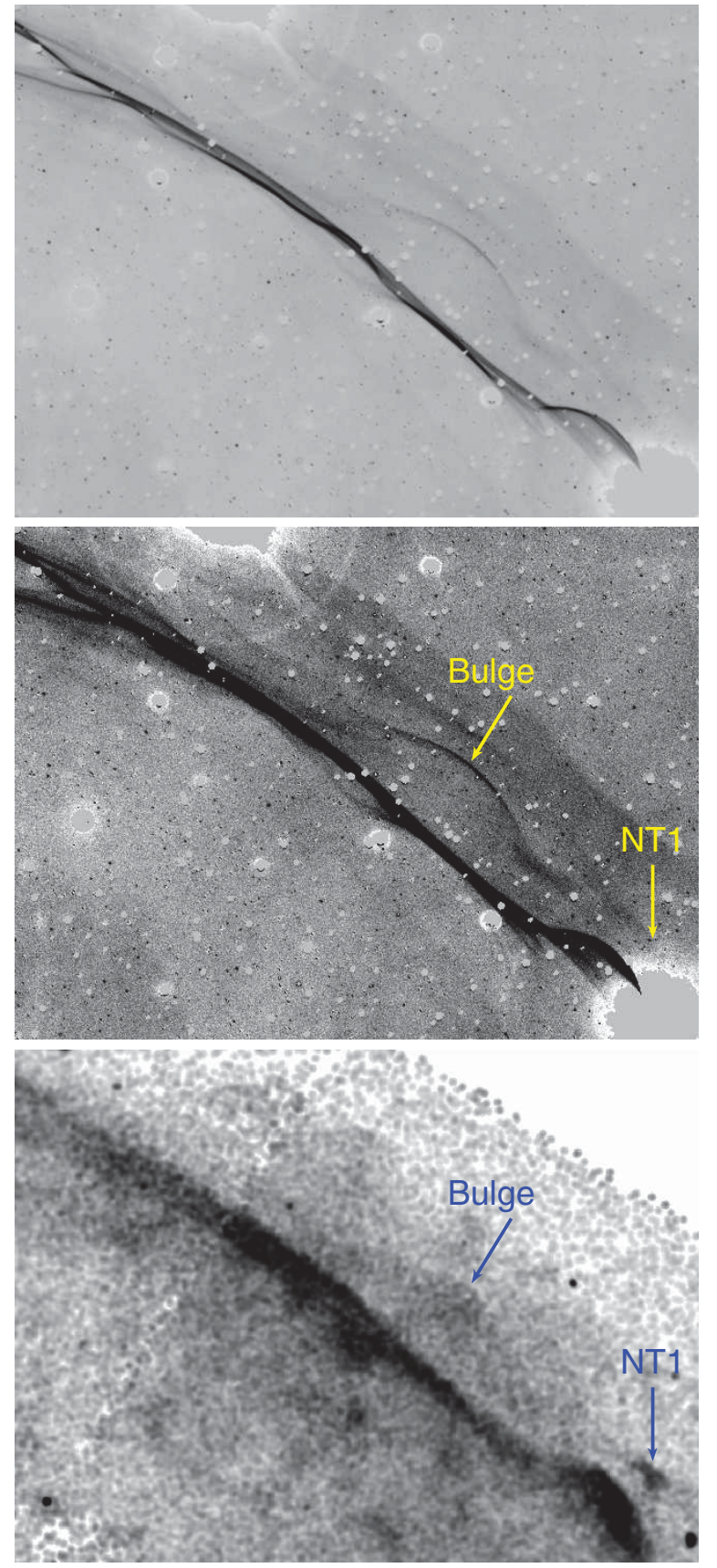

Figure 5. Same $10^{\prime} \times 7.5$ region along the NW shock is shown these images: the top and middle are in $\mathrm{H} \alpha$, displayed at different grayscale levels. The upper image shows the delicate bright filament, while the middle one shows much fainter emission farther out, indicating the complex three-dimensional structure of the shock front. The lower panel shows the soft $(0.5-1.2 \mathrm{keV}) \mathrm{X}$-ray image, displayed to show the coincident faint emission to the NW. The bulge ahead of the bright $\mathrm{H} \alpha / \mathrm{X}$-ray filament is easily seen in both $\mathrm{H} \alpha$ and X-rays. The feature marked NT1 is a nonthermal filament noted by Katsuda et al. (2013) whose proper motion indicates a velocity much higher than that for the brighter thermal filament in the NW; faint optical emission is also seen just ahead of this filament, though this is partially lost to the bright halo around a bright star in this region.

(A color version of this figure is available in the online journal.)

To study the expansion around the entire shell, we have used as the first-epoch image that obtained from a set of eleven overlapping ACIS-I exposures from 2003 (PI: J. Hughes; Cassam-Chenaï et al. 2008), each with an exposure time of about $20 \mathrm{ks}$. We reprocessed this data set using the same CIAO and

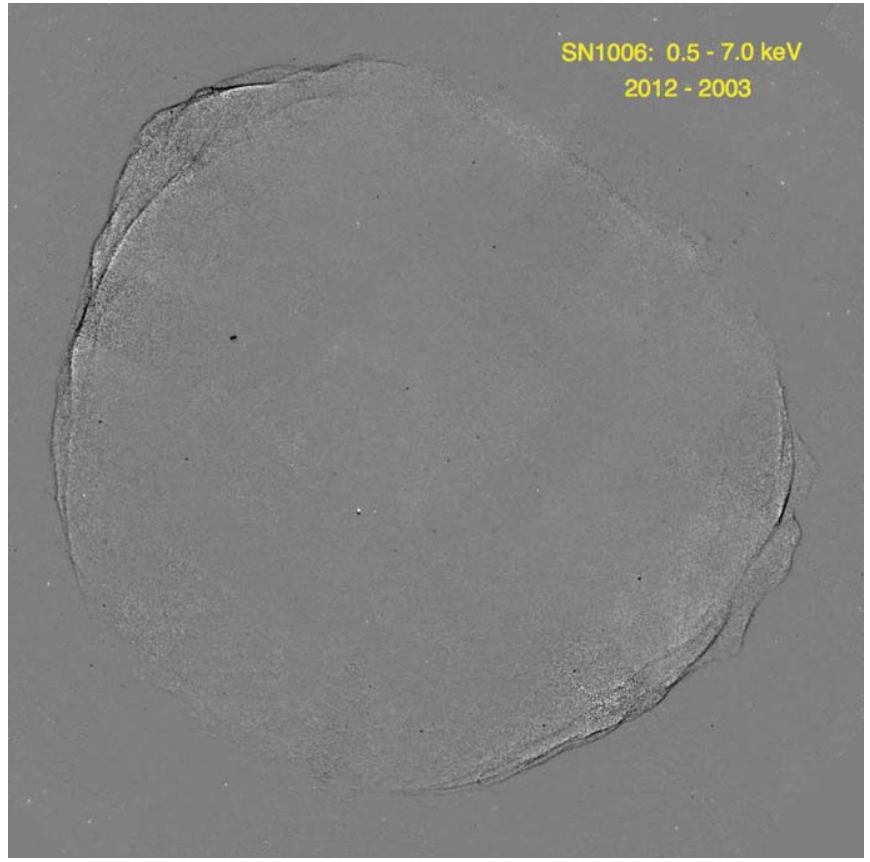

Figure 6. Difference image between the merged 2012 image (0.5-7.0 keV, the sum of all three bands from Figure 2, shown as black here) and the 2003 one (Cassam-Chenaï et al. 2008, shown as white). The 2003 data were aspectcorrected to match those from 2012, and then merged through a process identical to that for 2012. Expansion is especially evident along the sharp NE and SW limbs, but is noticeable around almost the entire perimeter. The point source that located just SE of the geometric center, which appears to have moved slightly southward, corresponds to a foreground star with high proper motion, and was not used in the image registration.

(An animation and a color version of this figure are available in the online journal.)

CALDB versions as for the 2012 data. In order to assure that the data from the two epochs were accurately registered, we began with the set of X-ray point sources located by wavdetect in each of the ten 2012 fields (after our small aspect corrections), and selected only those with significance $>6 \sigma$. For the many duplicate sources (the result of field overlap), we kept only the one with the smallest error ellipse in each case, to give a master list of 129 distinct sources. We then determined the small aspect correction for each of the eleven fields from 2003 by using wavdetect on each field and matching the resulting source list against our master list. (This procedure is preferable to registering the 2003 data using the NOMAD optical catalog — as with the 2012 data-because there are far more X-ray point sources than astrometric stars, and because fewer of those stars were detected with high significance in the shorter 2003 X-ray exposures.) Finally, we used merge_obs to combine the 2003 data, just as with that from 2012.

The structure of SN 1006 appears virtually identical in both data sets, though the deeper 2012 data reveal it in greater detail. By aligning and blinking the 2003 and 2012 images, however, expansion of the shell becomes obvious. The expansion is shown somewhat less dramatically in Figure 6, which is simply the difference between the merged images at the two epochs. The expansion is most obvious in the NE and SW, where the shock front is most sharply defined and the X-ray emission is brightest. While the expansion of other regions around the shell is less obvious in the difference image, it is clear on blinking the images, as in the animation that appears in the online version of Figure 6. 


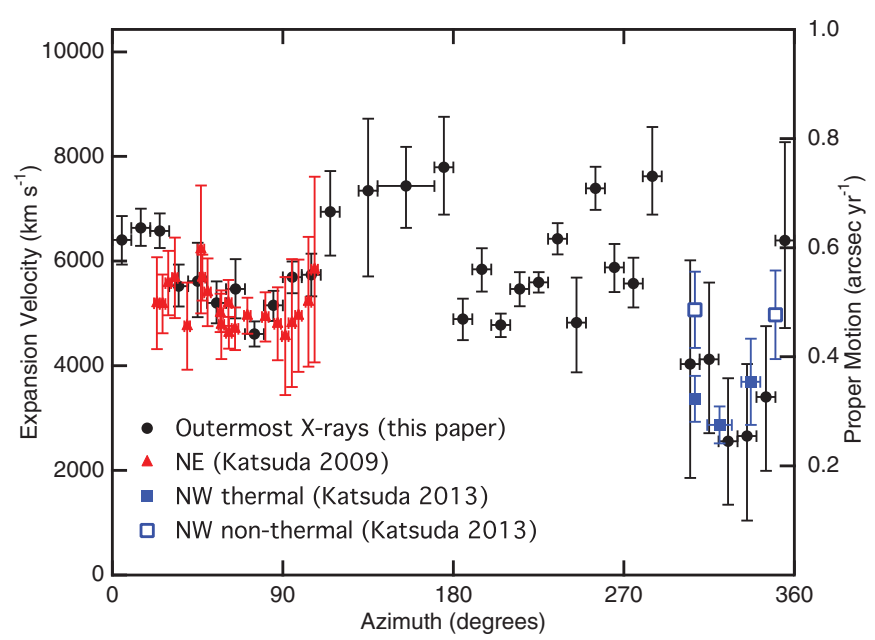

Figure 7. Black circular points show the measured expansion of the SN 1006 X-ray limb from 2003 to 2012, plotted as a function of azimuth (defined as counterclockwise from north). Also shown are the measurements based on individual features in the NE (red triangles, Katsuda et al. 2009) and the NW (in blue, Katsuda et al. 2013), where there are a few small non-thermal features (open squares) in addition to the thermal ones (filled squares). For the expansion velocity, we assume a distance of $2.2 \mathrm{kpc}$. All the uncertainties indicate $90 \%$ confidence limits.

(A color version of this figure is available in the online journal.)

In Figure 7 we show the measured proper motion as a function of azimuthal angle (measured in the conventional sense, rotating eastward from north). We took the center to be at R.A. $=15^{\mathrm{h}} 02^{\mathrm{m}} 54^{\mathrm{s}} .9$, decl. $=-41^{\circ} 56^{\prime} 08^{\prime \prime} .9$ (J2000.), the same as that defined by Katsuda et al. (2009), and extracted radial profiles in $10^{\circ}$ azimuthal sectors from the merged, aspectcorrected images for both 2003 and 2012. We then carried out a minimum- $\chi^{2}$ analysis limited to the outermost edge of clear X-ray emission to determine the shifts-in the purely radial direction, regardless of the orientation of individual features-between the two epochs, which are separated in time by 9.1 yr. (The 2003 observations were all carried out over 4 days, 2003 April 8-11. Those in 2012 were spaced over two months, from 2012 April 20 to 2012 June 15; we used the mean epoch.) The procedure is similar to that we used in earlier analyses of the NE and NW rims (Katsuda et al. 2009, 2013), except in those papers we identified and measured the proper motion for individual identifiable features, in a direction normal to the local shock surface, which in some cases was significantly nonradial. ${ }^{10}$ In order to provide consistency around the entire circumference, including those regions with no crisply defined features, in the present measurement we simply used the portion of the periphery that falls within an azimuthal sector, and measured the purely radial motion. We show typical profiles from both epochs, one from each of the four quadrants, in Figure 8. ${ }^{11}$

For the synchrotron-dominated regions in the NE and SW, where the shock is clearly defined in X-rays, the above method gives precise measurements, and for most of the NE these are in excellent agreement with those of Katsuda et al. (2009, also shown in Figure 7). Only in the $20^{\circ}-30^{\circ}$ sector is there a seeming

\footnotetext{
10 Another significant difference is that in both the NE and NW measurements, the first-epoch images were far deeper than the 2003 one used here.

11 In the NW, we made an exception to measuring the outermost edge of $\mathrm{X}$-ray emission: there the very faint emission beyond the bright filament, described in the previous section, is simply to faint to give a measurement. Instead, the measurement at azimuths about $310^{\circ}-350^{\circ}$ is really for the bright (thermal) X-ray filament. An example is shown in Figure 8, upper right.
}

disagreement, but examination of Figure 1 of that paper shows that the individual features measured by Katsuda et al. (2009) in the $20^{\circ}-30^{\circ}$ sector, while the brightest at this azimuth, do not lie at the outer edge, and are oriented far from normal to the radial direction. Thus, there is really no disagreement with earlier measurements in the NE.

In most of the thermal-dominated southeast (SE) quadrant, and in part of the NW, the shock front is not well defined in X-rays. Three sectors in the SE $\left(140^{\circ}-170^{\circ}\right)$, where the signal-to-noise was low and the individual measurements highly uncertain, were combined into a single $30^{\circ}$ sector. In the NW, comparison with the results from Katsuda et al. (2013, also shown in the figure) shows that some of these sectors include parts of both thermal and nonthermal features with quite different shock velocities, so the fact that profiles for the entire sector at different epochs did not correspond closely is hardly surprising. For two sectors, centered at azimuths $125^{\circ}$ and $295^{\circ}$, there was not a sufficiently sharp X-ray limb to yield a proper-motion measurement at all. All of the measurements shown in Figure 7 gave satisfactory fits (reduced $\chi^{2} \sim 1$ ), and there were no systematic differences in $\chi^{2}$ as a function of azimuth, post-shock brightness, or measured velocity. In addition, we examined each fit by eye to ensure that all look reasonable.

The most notable fact about the proper-motion measurements is that the expansion velocity in the $\mathrm{SE}$ is higher than anywhere else around the shell: $\sim 7400 \pm 800 \mathrm{~km} \mathrm{~s}^{-1}$, almost 2.5 times faster than that of the far brighter thermal X-ray filament in the NW. In the SE, the shock front is not really defined at all in $\mathrm{X}$-rays (Figure 2); instead the outermost emission is marked by tufts that we interpret as SN ejecta based both on their kinematics and their spectra (see Section 6). Furthermore, some of these tufts are located beyond the outermost of the multiple indistinct shells seen in $\mathrm{H} \alpha$ (Figure 3).

A common way of expressing the proper motion in an SNR is through the expansion parameter $m$ : the power-law index in $R \propto t^{m}$, where $t$ is the age of the remnant. This parameter can be interpreted as the ratio of current expansion rate divided by the mean rate over the remnant's lifetime, $m=\mu t / \theta$, where $\theta$ is the angular radius. The outer tufts of emission in the SW are located $\sim 14^{\prime} .8=9.5 \mathrm{pc}$ from the center, so with an age of $1001.5 \mathrm{yr}$ (the mean for the 2003 and 2012 epochs) we find $m=0.80 \pm 0.08$, close to the free-expansion value of 1 . This value contrasts sharply with that of $m=0.54 \pm 0.05$ measured by Katsuda et al. (2009) for the nonthermal NE shell, which suggested that in the NE SN 1006 is transitioning to the adiabatic phase ( $m \sim 0.4)$. It is entirely consistent to interpret the tufts that define the SE X-ray periphery of SN 1006 as plumes of ejecta that have been coasting almost undecelerated into a very low-density region of the interstellar medium (ISM). The proper motions we have measured for these tufts do not represent a shock velocity, but rather the current motions for these ejecta tufts.

Simulations of either ejecta bullets originating in the SN explosion (e.g., Orlando et al. 2012) or R-T "mushroom caps" that formed more recently from hydrodynamic instabilities (e.g., Warren \& Blondin 2013) show that denser regions can move faster than the blast wave. Ejecta "bullets" overtake the blast wave, move beyond its mean radius briefly (as in knots D and $\mathrm{E}$ in Figure 13), and are shredded and dissipate. R-T "mushrooms" also represent regions of denser-than-average ejecta, which formed much later but which can also, for highly compressive shocks, penetrate the forward shock before they die 

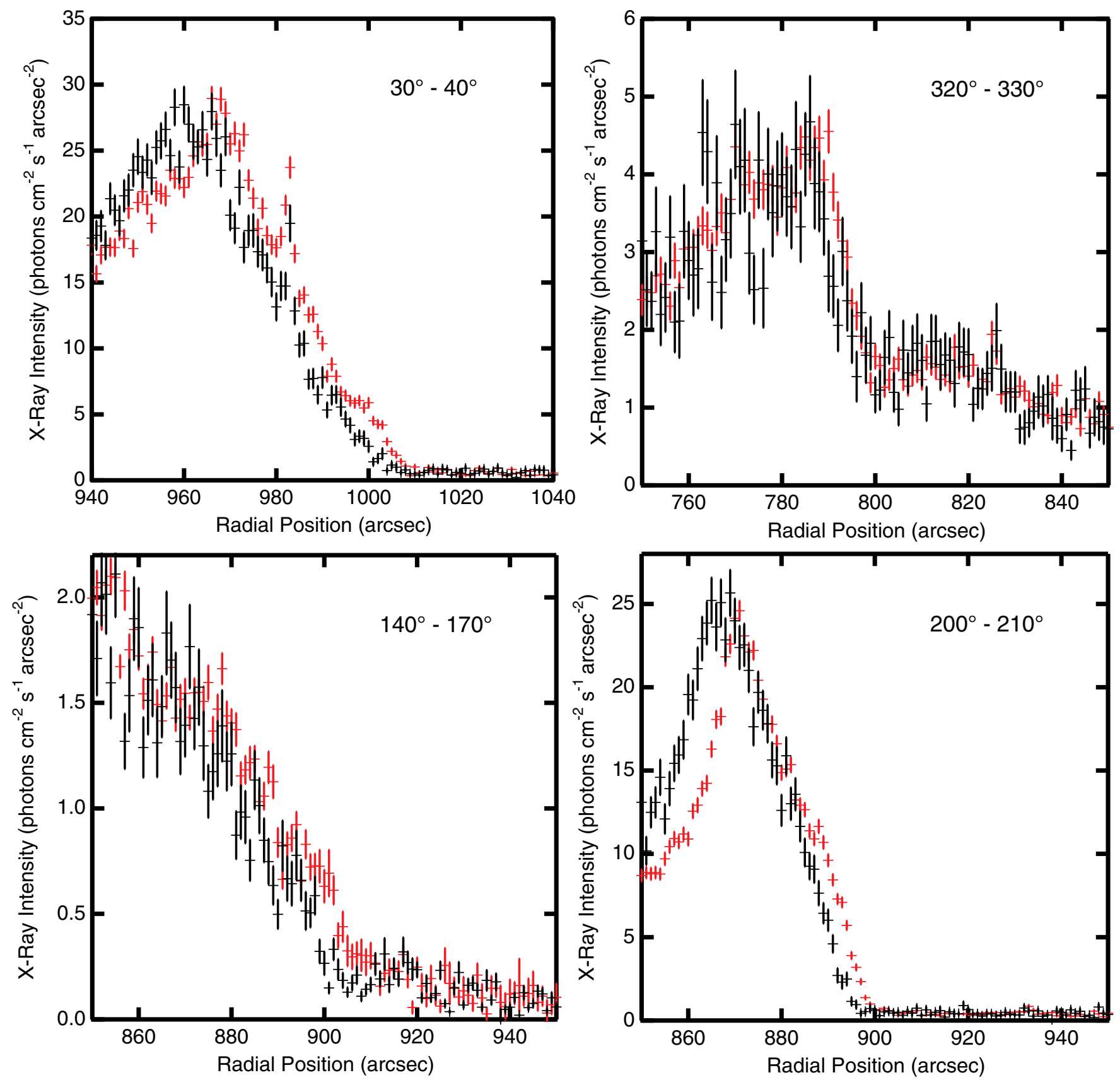

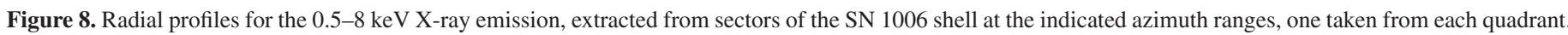

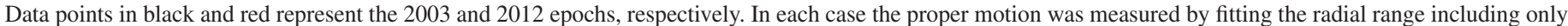
the outermost X-ray emission.

(A color version of this figure is available in the online journal.)

back. It is, therefore, not surprising that the ejecta plumes in the SE currently show higher velocities than the average blast wave around most of SN 1006.

Velocities around most of the synchrotron-dominated NE and SW limbs center around $5000 \mathrm{~km} \mathrm{~s}^{-1}$. (Exceptions include the "bulge" to the north, azimuths $\sim 10^{\circ}-30^{\circ}$, that shows a currently higher velocity than adjacent regions, and a point at about $260^{\circ}$ that may reflect the "bulge" apparent in the west-SW.) Variations in the velocities are a bit larger in percentage terms than variations in the remnant radius, implying that the upstream ISM density varies both azimuthally around the original SN location and with distance from it, as is most clearly demonstrated in the NW.

\section{X-RAY SPECTRA: THERMAL EMISSION FROM SUPERNOVA EJECTA AND THE ISM}

In order to investigate the spatial distribution of emission from different elements stemming from $\mathrm{SN}$ ejecta or from the ISM, we have produced equivalent-width (EW) images in characteristic lines, according to the following procedure, similar to that introduced by Hwang et al. (2000) for Cas A. We first extracted images, binned by a factor of eight (4" pixels) in a number of narrow energy bands corresponding to K-line emission from significant elements: $\mathrm{O}, \mathrm{Ne}, \mathrm{Mg}$, and $\mathrm{Si}$, and also in narrow line-free continuum bands to either side of each of the line image (all the bands are detailed in Table 3). We 

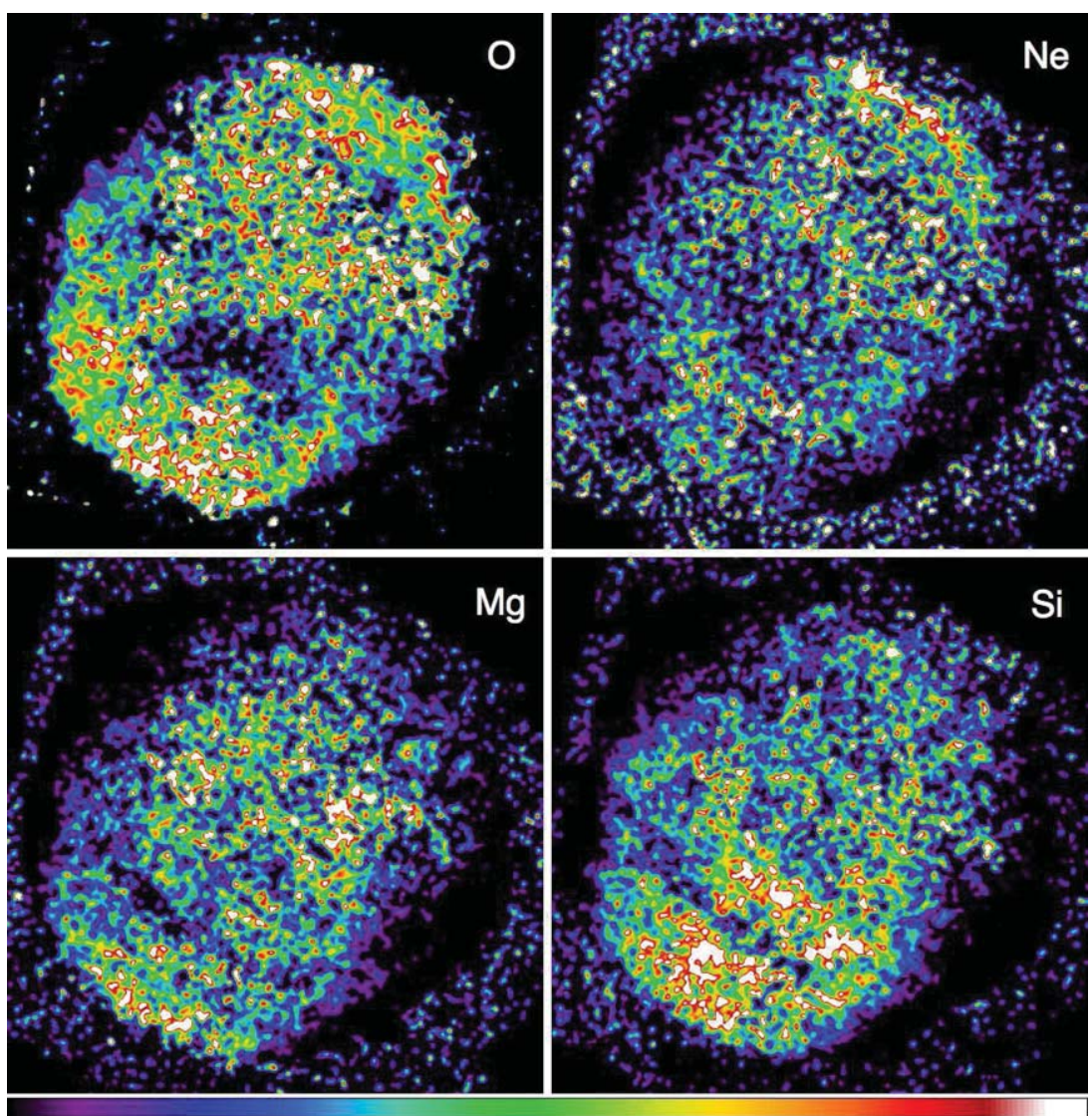

Figure 9. Equivalent-width images in the $\mathrm{K}$ lines of $\mathrm{O}, \mathrm{Ne}, \mathrm{Mg}$, and $\mathrm{Si}$, produced according to the procedure described in the text. The intensity scales are linear, starting from 0 in each case with a maximum of $1 \mathrm{keV}(\mathrm{O}), 0.12 \mathrm{keV}(\mathrm{Ne}), 0.25 \mathrm{keV}(\mathrm{Mg}), 0.6 \mathrm{keV}(\mathrm{Si})$. The field is $36^{\prime}$ square, oriented north up, east left. An excess of line emission in the SE quadrant is evident in Si especially — an indication of an asymmetry in the ejecta distribution. Virtually no line emission is seen from the crescent-shaped regions on the NE and SW limbs, since these regions are completely dominated by nonthermal emission.

Table 3

Equivalent Width Energy Bands

\begin{tabular}{lccc}
\hline \hline Element & $\begin{array}{c}\text { Line Energy } \\
(\mathrm{keV})\end{array}$ & $\begin{array}{c}\text { Cont. } \\
(\text { Low, keV })^{\mathrm{a}}\end{array}$ & $\begin{array}{c}\text { Cont. } \\
(\mathrm{High}, \mathrm{keV})^{\mathrm{a}}\end{array}$ \\
\hline Oxygen & $0.51-0.74$ & $0.4-0.51$ & $0.74-0.87$ \\
Neon & $0.89-0.97$ & $0.74-0.87$ & $1.12-1.2$ \\
Magnesium & $1.29-1.42$ & $1.20-1.29$ & $1.42-1.7$ \\
Silicon & $1.7-1.95$ & $1.42-1.7$ & $1.95-2.2$ \\
\hline
\end{tabular}

Notes. ${ }^{\text {a }}$ Cont. (Low) and Cont. (High) give the continuum bands on either side of the line energy, used in producing the EW images as described in the text.

then smoothed all the images slightly with a 2 pixel Gaussian filter, divided each continuum image by its bandwidth in $\mathrm{keV}$, logarithmically interpolated between high and low continuum bands, and subtracted the appropriate continuum from each emission-line image. Finally, to better distinguish between composition and density effects, we divided each continuumsubtracted image by the appropriate continuum one to produce the EW images. These are analogous to optical EW images, except that in our case the units are $\mathrm{keV}$ instead of $\AA$.

The resulting images are shown in Figure 9. In all four images the NE and SW limbs appear dark, indicating little line emission, since strong synchrotron radiation there dominates any thermal emission. Within the interior, however, there are distinct differences. Silicon, expected to stem primarily from the ejecta in a Type Ia SN, is strongly concentrated in the SE quadrant-suggesting a clear asymmetry in either the distribution of Si ejecta or in the (presumably reverse) shock pattern that has heated it. This confirms the recent Suzaku result from Uchida et al. (2013). Oxygen and magnesium show less extreme concentrations in the SE, and also concentrations well inside the shell rim to the NW. These too probably arise largely from SN ejecta, with significant contributions from the shocked ISM. Oxygen in particular is also strong behind the primary shock to the NW. ${ }^{12}$

Neon is also prominent in the NW, but strongly concentrated in a narrow filament immediately behind the shock front (Figure 9). Given the morphology of the Ne filament and its location where both the pre-shock density is highest (e.g., Winkler et al. 2013 and references therein) and the overall thermal emission is strongest (Figure 2), it seems certain that this feature arises from shocked ISM. Elsewhere within SN 1006, however, the Ne emission is likely a mixture of shocked ISM and ejecta. The distribution of Ne within the shell most closely resembles that of $\mathrm{Mg}$, which, like $\mathrm{Ne}$, results from Carbonburning. One curious feature in the $\mathrm{Ne}$ distribution is the relatively strong band curving from about nine o'clock to five o'clock across the SE quadrant. Both $\mathrm{Mg}$ and $\mathrm{Si}$ emission are relatively weak along this same band; the cause of these effects remains under investigation.

In order to further investigate the contrasts that are evident in Figure 9, we have selected the brightest $25 \%$ of the pixels in

\footnotetext{
${ }^{12}$ Also important in the ejecta from SN Ia is Fe, whose $\mathrm{K}$-lines at $6.7 \mathrm{keV}$ are shown clearly by Uchida et al. (2013). However, the ACIS sensitivity above $5 \mathrm{keV}$ is too low to enable significant measurements in the faint thermal plasma of SN 1006
} 

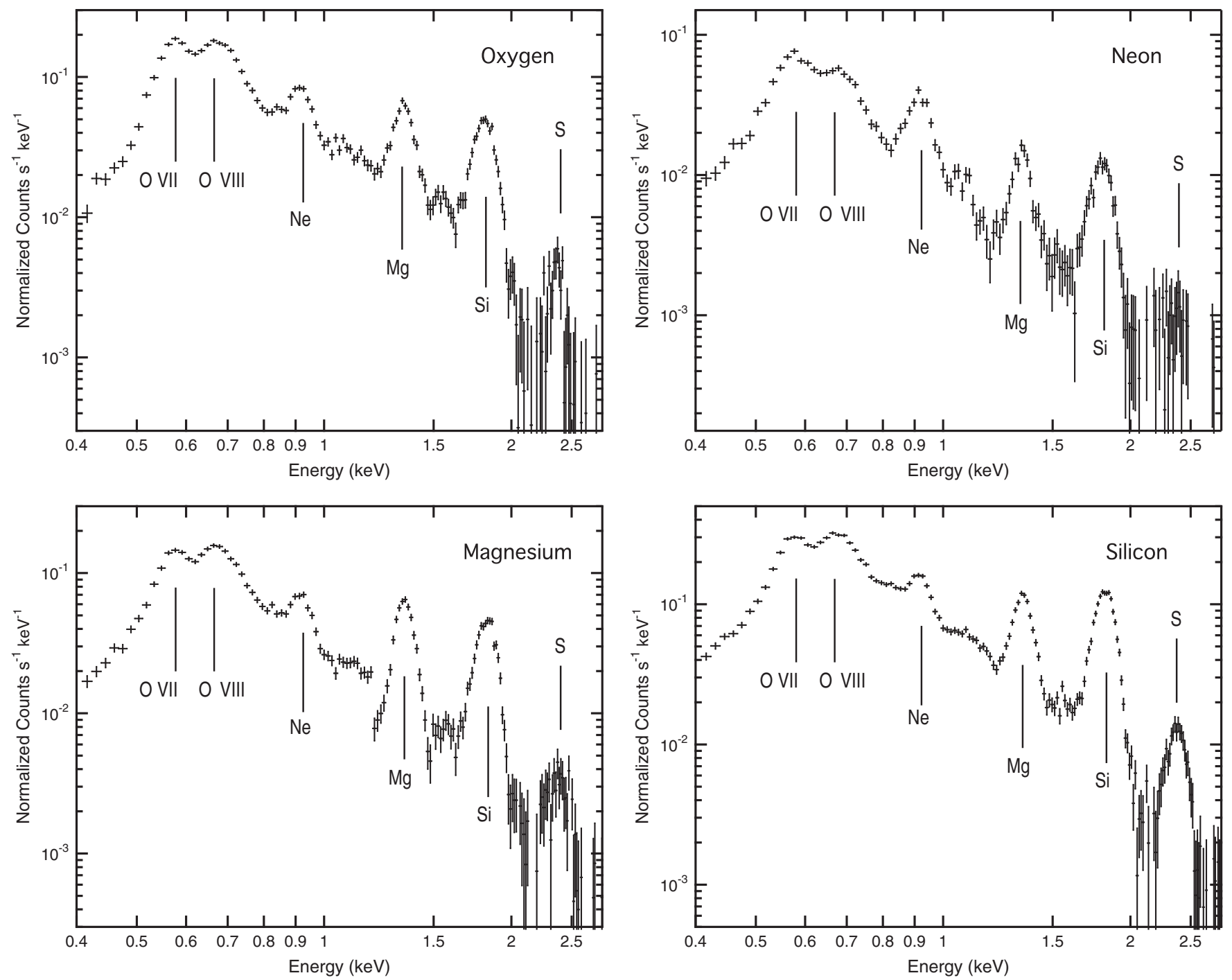

Figure 10. X-ray spectra extracted from the brightest $25 \%$ of the pixels in each of the equivalent-width images (Figure 9 ).

each of the EW images, and extracted a combined spectrum from these, with the results shown in Figure 10. These regions are not mutually exclusive; e.g., the brightest $25 \%$ of the Si pixels include some of the brightest $25 \%$ of the Mg pixels, etc. A few interesting trends can be seen in comparing these spectra. While all of the spectra clearly contain significant emission from ejecta, the "Ne-pixel" spectrum is heavily weighted by pixels along the bright NW shock, and hence should emphasize emission from forward-shocked ISM. (While some ejecta may contribute, especially from Ne-bright pixels in the interior, nucleosynthetic models, e.g., Nomoto et al. 1984; Iwamoto et al. 1999; Maeda et al. 2010, predict far less Ne than either O or Si, and somewhat less than $\mathrm{Mg}$, in SN Ia ejecta.) And indeed this spectrum, in addition to showing a Ne IX $0.92 \mathrm{keV}$ line that is much stronger (relative to the lines from other elements) than the others in Figure 10, also shows $\mathrm{O}$ lines with significantly stronger He-like $(0.57 \mathrm{keV})$ than $\mathrm{H}$-like $(0.65 \mathrm{keV})$ ions. These $\mathrm{O}$ lines indicate that the ionization state of the gas is lower than in the ejecta-dominated regions, consistent with a spectrum dominated by recently shocked ISM.

To better understand the spectra resulting from these "brightest pixels" in the EW images, we have modeled each of the spectra with an identical model in XSPEC. We used a single absorbed, variable-abundance, non-equilibrium ionization model (phabs*vnei), with the absorption column frozen for all spectra to a value of $7 \times 10^{20} \mathrm{~cm}^{-2}$. The temperature and ionization state of the plasma were allowed to vary, as were the abundances of the four elements represented in Figure 10: $\mathrm{O}, \mathrm{Ne}, \mathrm{Mg}$, and $\mathrm{Si}$. We fit the spectra only up to $2 \mathrm{keV}$. We stress here that these models are not intended to be "physical" models that accurately represent the current conditions of the plasma. Such a model would be highly complex, since each of these four spectra represent an amalgam of spectra from physical locations all over the remnant, and all undoubtedly contain emission from both forward-shocked ISM and reverse-shocked ejecta. (This complexity is demonstrated by the multiple components required to fit the Suzaku spectra; Yamaguchi et al. 2008; Uchida et al. 2013.) Rather, we have simply extracted qualitative differences between the spectra, primarily in terms of the relative abundances implied by the model fits. ${ }^{13}$

\footnotetext{
13 Other effects may play a role as well: the $0.74-0.87 \mathrm{keV}$ band, which we use as a continuum for both the $\mathrm{O}$ and $\mathrm{Ne} \mathrm{EW}$ images, includes a "false continuum" produced by blended Fe-L lines, so an anomalously low $\mathrm{Fe}$ abundance may artificially increase the $\mathrm{O}$ and $\mathrm{Ne}$ EW. A similar effect for the $\mathrm{O}$ images only may be produced from anomalous $\mathrm{N}$ abundance, since the low continuum for the $\mathrm{O}$ EW images includes lines from N. Finally, temperature variations can also affect the EW values. Detailed discussion of these issues is beyond the scope of this initial report.
} 


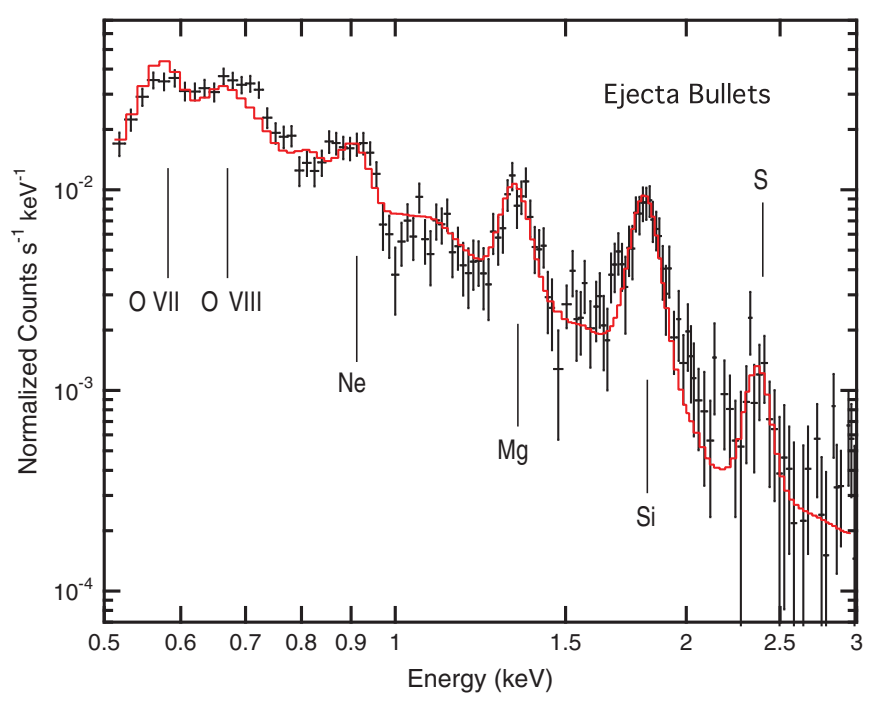

Figure 11. Combined spectrum of the two ejecta bullets shown in Figure 4, with local background subtracted. The best fit with an absorbed VNEI model is shown in red.

(A color version of this figure is available in the online journal.)

The model fits confirm that the EW images are indeed showing meaningful variations in the abundances of the respective metals. For instance, the ratio of $\mathrm{Si} / \mathrm{Ne}$ is approximately twice as high in the Si-selected spectrum of Figure 10 compared to the $\mathrm{Ne}$ one, and the $\mathrm{O} / \mathrm{Ne}$ ratio is $70 \%$ higher in the $\mathrm{O}$-selected spectrum compared to the $\mathrm{Ne}$ one-with small formal errors in both cases. ${ }^{14}$ Our spectra show that while the broadband image of SN 1006 may appear relatively uniform in the interior, one can still use regions of strong $\mathrm{Si}$ and $\mathrm{S}$, at least, to identify regions dominated by ejecta.

Finally, we have extracted a combined spectrum from the two ejecta "bullets" preceded by $\mathrm{H} \alpha$ bowshocks shown in Figure 4, subtracted a local background from several diffuse regions nearby, and have fit this with the same phabs*vnei model in XSPEC. The result, shown in Figure 11, requires strong overabundances of $\mathrm{Si}(\sim 6$ times solar $)$ and $\mathrm{S}(\sim 11$ times solar $)$, and an underabundance of $\mathrm{Ne}(\sim 0.2$ times solar $)$. The paucity of counts in these spectra, together with the problem of the bullets being superimposed on the more diffuse background, limit the quantitative validity of the fits, but there can be little doubt that they are, indeed, composed primarily of ejecta.

\section{A SHOCK PRECURSOR?}

A firm prediction of diffusive shock acceleration theory is that accelerated electrons will spend some of their time ahead of the shock, producing synchrotron radiation in a "halo" of $\mathrm{X}$-ray emission. A precursor X-ray halo has yet to be conclusively identified in any young SNR, but SN 1006, which is nearby, with low foreground absorption and well-defined synchrotron rims, probably presents the best opportunity to detect one. As we discuss below, two potential observables for a precursor halo are the spatial extent and the magnitude of the sharp jump at the shock front.

Long et al. (2003) used Chandra observations from 2001 to search for halo emission in the NE region, and found that

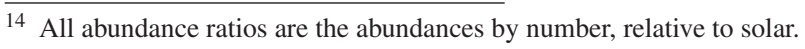

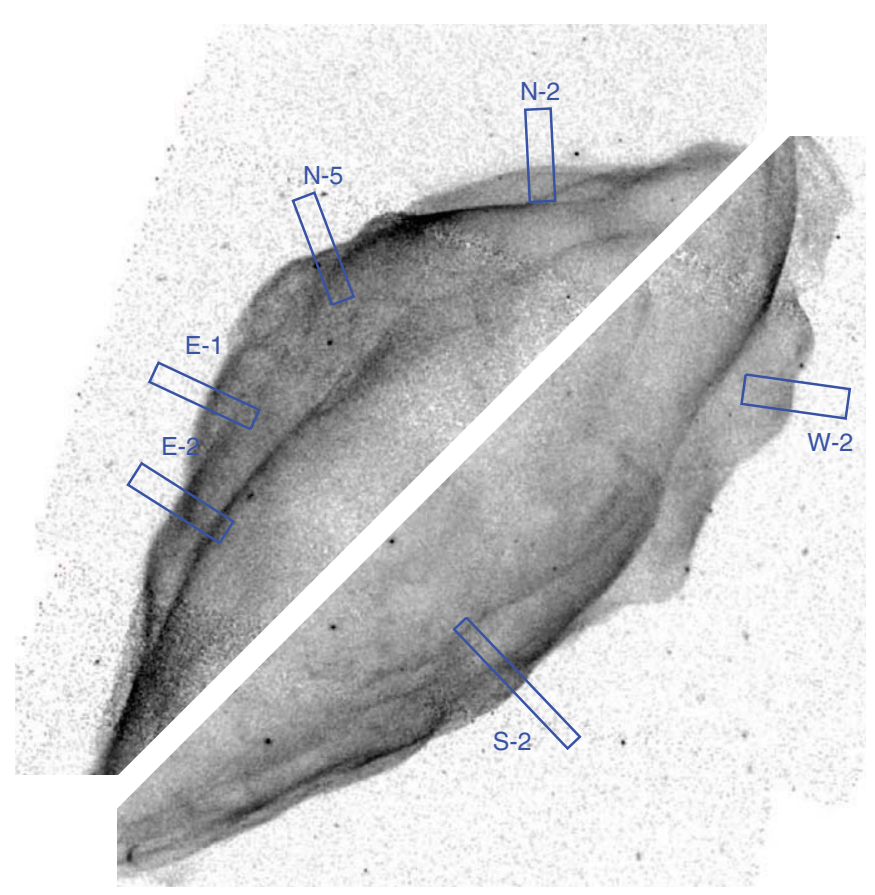

Figure 12. Composite showing the NE (upper left) and SW (lower right) synchrotron-dominated limbs in the $0.5-7 \mathrm{keV}$ mosaic image of SN 1006. The blue rectangles indicate regions where the precursor profiles plotted in Figure 13 were taken.

(A color version of this figure is available in the online journal.)

any halo is very faint, with a mean surface brightness $\lesssim 1.5 \%$ of the peak surface brightness at the shock front, or is very thin, limited in extent to under a few arcseconds ahead of the shock. Subsequently, Morlino et al. (2010) described a model in which pre-shock emission rises steeply prior to the shock over a scale of $\sim 10^{\prime \prime}$, followed by a final jump by an order of magnitude to reach the peak emission-due to a magnetic field jump of a factor of $\sim 4$ at the "viscous subshock" (see below). They found this model halo to be consistent with profiles observed in the NE by Long et al. (2003), and also with the 2008 epoch observation of the same region (Katsuda et al. 2009).

\subsection{New Observational Results}

Here, we report a similar analysis to that of Long et al. (2003), but carried out in greater detail over a larger sample of regions of interest. We have selected six regions shown in Figure 12: four along the NE limb and two along the SW. All of the regions are located in places where the local shock front is nearly linear, and are $20^{\prime \prime}-40^{\prime \prime}$ wide (depending on the length of a clean segment of the rim), oriented perpendicular to the front. (Our region E-1 is similar to the one chosen by Morlino et al. 2010). In each case we selected the longest single observation for which each region was closest to on-axis, replaced any obvious point sources with local background, and then extracted profiles perpendicular to the shock front, to give the results shown in Figure 13. We used unbinned exposure-corrected flux images from $1-4 \mathrm{keV}$, eschewing both low- and high-energy emission to minimize background contamination. For each profile in the figure we also show the response expected from a sharp edge of emission, folded through the point-spread function (PSF) applicable at the matching location on the ACIS detectors. For consistency in the figure, we have normalized the post-shock peak (determined 

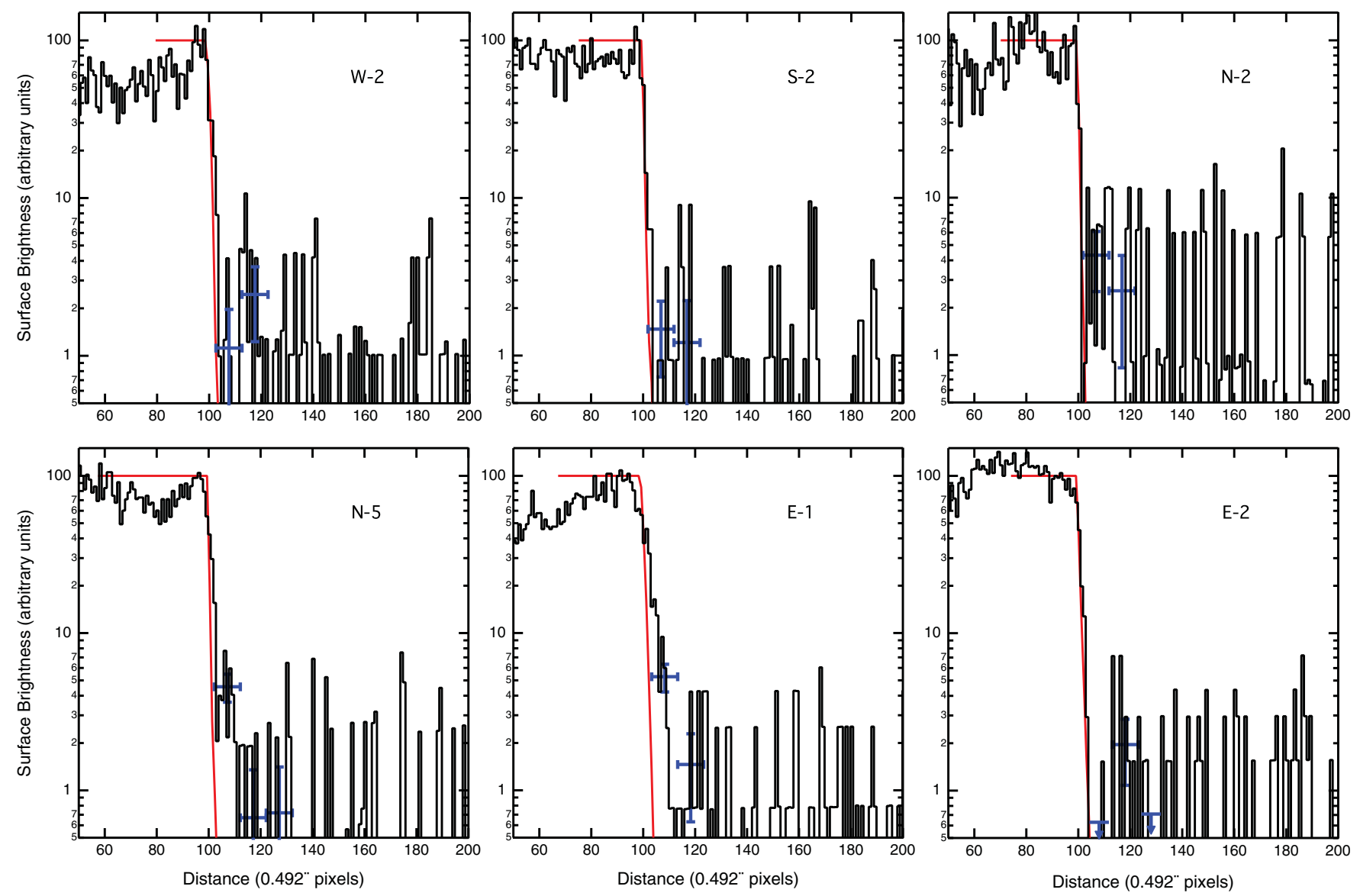

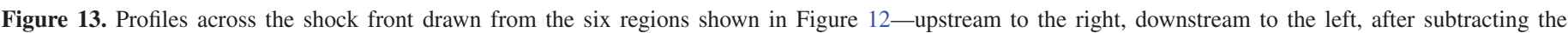

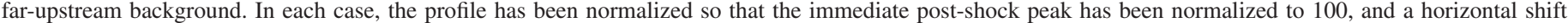

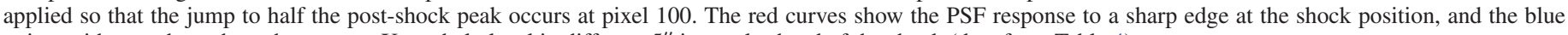
points with error bars show the average X-ray halo level in different $5^{\prime \prime}$ intervals ahead of the shock (data from Table 4).

(A color version of this figure is available in the online journal.)

after smoothing with a Gaussian of FWHM $\approx \mathrm{PSF}$ ) to a common level, and have shifted each profile in the horizontal direction so that the rise to $50 \%$ of the peak occurs exactly at pixel 100 .

To give a quantitative measure for a putative halo, we have measured the surface brightness averaged over three narrow angular ranges ahead of the shock: $0^{\prime \prime}-5^{\prime \prime}, 5^{\prime \prime}-10^{\prime \prime}$, and $10^{\prime \prime}-15^{\prime \prime}$. For the zero point, we have taken the halfheight point of the measured profile and have added the distance over which the appropriate PSF drops from its peak to $10 \%$ of its peak value. For the local background, we have measured the average level starting 20" upstream from the shock and extending to $50^{\prime \prime}$. These measurements were carried out on the exposure-corrected, flux-calibrated images (units photons $\mathrm{cm}^{-2} \mathrm{~s}^{-1}$ pixel $^{-1}$ ); to obtain the uncertainties, we extracted profiles from the identical regions in the raw counts images and obtained the relative uncertainties directly from Poisson statistics. The results are given in Table 4, and are also indicated in Figure 13. In the table, the post-shock peak value is that of the immediate post-shock peak (again after smoothing using a Gaussian with FWHM $\approx$ PSF), and the peak/halo value is the ratio of this value to that of the net halo averaged over $0^{\prime \prime}-5^{\prime \prime}$.

In five of the six cases we find a small excess over the local background for the X-ray flux $0^{\prime \prime}-5^{\prime \prime}$ ahead of the shock, though the statistical significance is low in all but two of the cases.
From that level, the X-ray flux jumps by well over an order of magnitude, over a distance comparable to the PSF width of $2^{\prime \prime}-4^{\prime \prime}$, to the immediate post-shock peak. Further upstream from the shock front, in the range $5^{\prime \prime}-10^{\prime \prime}$, the excess over background has dropped to below $2 \sigma$ (except for region E-2, the one which showed no excess over $0^{\prime \prime}-5^{\prime \prime}$ ), and beyond $10^{\prime \prime}$ the flux is essentially indistinguishable from the background level in all six regions. The region that shows the greatest evidence for a halo is E-1, which is similar to that used by Morlino et al. (2010), and which is also the one where the observation was made farthest off-axis. Here the flux jumps across the shock by a factor $\sim 20$, and the pre-shock emission appears to follow nearexponential decay ahead of the shock, falling off by $1 / e$ in about $4^{\prime \prime} \approx 1.3 \times 10^{17} \mathrm{~cm}$. In the other cases, the decay scale is shorter, and/or the jump from halo to post-shock peak is greater. We point out that there are a number of factors other than a true halo that could give faint emission ahead of the peak: curvature across the region, projection effects along the line of sight, faint point sources that were not excised, and/or intrinsic PSF response; yet there is no plausible way to make the shock jump appear sharper than it really is. While we can by no means rule out the existence of a precursor halo, a fair summary of our results is that halo emission in the $1-4 \mathrm{keV}$ range is typically narrower than $3^{\prime \prime}$, and that across the shock the emission typically jumps by at least a factor of 20 . 
Table 4

Precursor Measurements

\begin{tabular}{|c|c|c|c|c|c|c|c|}
\hline \multirow[t]{2}{*}{ Region } & \multirow[t]{2}{*}{ ObsID } & \multirow{2}{*}{$\begin{array}{c}\text { Post-shock } \\
\text { Peak }^{\mathrm{a}}\end{array}$} & \multirow{2}{*}{$\begin{array}{c}\text { Background }^{\mathrm{a}} \\
20^{\prime \prime}-50^{\prime \prime} \\
\end{array}$} & \multicolumn{3}{|c|}{ Net Pre-shock Halo ${ }^{\mathrm{a}, \mathrm{b}}$} & \multirow{2}{*}{$\begin{array}{c}\text { Ratio }^{c} \\
\text { Peak/Halo }\left(0^{\prime \prime}-5^{\prime \prime}\right)\end{array}$} \\
\hline & & & & $0^{\prime \prime}-5^{\prime \prime}$ & $5^{\prime \prime}-10^{\prime \prime}$ & $10^{\prime \prime}-15^{\prime \prime}$ & \\
\hline W-2 & 9107 & 206 & $4.5 \pm 0.6$ & $2.3 \pm 1.7$ & $5.1 \pm 2.5$ & $0.3 \pm 1.6$ & 89 \\
\hline S-2 & 13739 & 312 & $5.5 \pm 0.9$ & $4.6 \pm 2.3$ & $3.8 \pm 3.2$ & $-1.3 \pm 1.6$ & 68 \\
\hline $\mathrm{N}-2$ & 13743 & 125 & $5.4 \pm 0.6$ & $5.3 \pm 2.2$ & $3.2 \pm 2.2$ & $0.6 \pm 1.7$ & 23 \\
\hline $\mathrm{N}-5$ & 13743 & 390 & $6.9 \pm 0.7$ & $17.8 \pm 3.6$ & $2.6 \pm 2.6$ & $2.8 \pm 2.7$ & 22 \\
\hline E-1 & 13738 & 461 & $12.5 \pm 1.0$ & $24.4 \pm 4.9$ & $6.8 \pm 3.8$ & $1.9 \pm 3.1$ & 19 \\
\hline E-2 & 9107 & 463 & $12.4 \pm 0.9$ & $-0.1 \pm 2.3$ & $9.1 \pm 3.5$ & $0.0 \pm 2.9$ & $>200$ \\
\hline
\end{tabular}

Notes.

a Surface brightness $\left(10^{-10}\right.$ photons $\mathrm{cm}^{-2} \mathrm{~s}^{-1}$ pixel $\left.^{-1}\right)$.

${ }^{\mathrm{b}}$ Measured surface brightness (after background subtraction) in indicated range ahead of shock.

c Surface brightness ratio: peak/net halo.

\subsection{Shock Models: Unmodified and Modified by Cosmic Rays}

For an unmodified shock, in which the pressure of upstream accelerated particles is negligible, we expect a sudden density jump by a factor of the compression ratio (four for a nonrelativistic monatomic gas) at the shock over a distance of a few thermal proton gyroradii, or about $5 \times 10^{9}(d / 2.2 \mathrm{kpc})\left(v_{\text {shock }} / 5000 \mathrm{~km} \mathrm{~s}^{-1}\right)(B / 10 \mu \mathrm{G})^{-1} \mathrm{~cm}$ for $\mathrm{SN}$ 1006. Upstream, the relativistic particles will diffuse ahead of the shock a distance of order $\kappa / v_{\text {shock }}$, where $\kappa$ is the diffusion coefficient (which may depend on particle energy as well as orientation with respect to the magnetic field). The flow velocity in the shock frame is constant at the upstream value until suddenly dropping due to viscous dissipation in the shock layer. This viscous dissipation heats the thermal gas and produces the obvious remnant edge. A precursor halo of X-ray emission would result from the upstream relativistic particles radiating in the presumably constant magnetic field.

But for the case of a cosmic-ray modified shock, where efficient shock acceleration produces a non-negligible pressure from fast particles, the gross dynamics are changed: the inflowing gas (in the shock frame) gradually slows over the diffusive scale length, $\kappa / v_{\text {shock}}$. As a result, the overall gas compression rises gradually over that length scale, rather than abruptly, although a final sharp jump at a remaining "viscous subshock" is expected. ${ }^{15}$ For a modified shock, the region in which the gradual deceleration and compression takes place is the shock precursor. In this region, tangential components of magnetic field would grow simply due to compression. In addition, the field can be amplified by large factors due to nonlinear effects (e.g., Bell 2004). All these factors contribute to the prediction of an X-ray synchrotron "halo" ahead of the viscous subshock (Reynolds 1996). Figure 14 illustrates these basic components in schematic form.

The expected scale length for any pre-shock emission would be somewhere between the (unrealistic) minimum of the electron gyroradius $r_{g}$ for an unmodified, perpendicular shock (without cross-field diffusion), and the diffusive scale length $\kappa / v_{\text {shock }}$ for a parallel shock. (Here "perpendicular" and "parallel" refer to the angle between the mean upstream

\footnotetext{
15 While some theoretical models predict that the viscous subshock will vanish altogether when particle acceleration is highly efficient, its presence in SN 1006 and other young remnants is clear from the existence of hot thermal plasma. Furthermore, typical calculations, such as Ellison et al. (1996) tend to find a minimum subshock compression ratio of about 2.5 as part of an overall larger compression ratio that can be quite large.
}

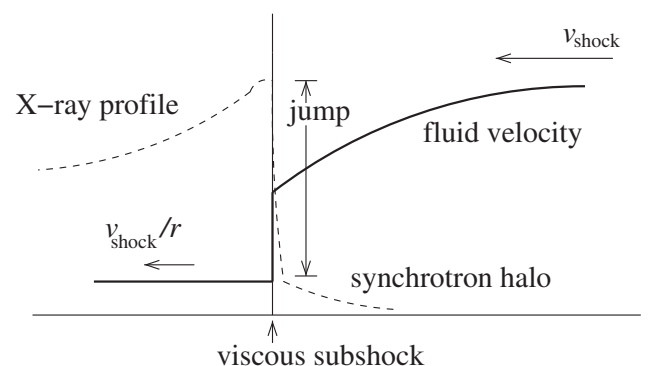

Figure 14. This schematic illustrates the geometry (in the frame of the shock) for a cosmic-ray modified shock. Gas flows in from the right with pre-shock velocity $v_{\text {shock}}$, and is gradually slowed before a sudden compression and deceleration at the viscous subshock. The compressed gas then flows out to the left, with velocity $v_{\text {shock }} / r$, where $r$ is the compression ratio. A precursor $\mathrm{X}$-ray halo would extend into the region ahead of the viscous subshock where deceleration is taking place.

magnetic field and the shock normal.) For a relativistic electron, $r_{g}=e E / B$, and such an electron emits the peak of its synchrotron radiation at an X-ray energy $h \nu_{0}=7.5 E^{2} B \mathrm{keV}$, giving $r_{g}=2.4 \times 10^{16}\left(h v_{0} / \mathrm{keV}\right)^{1 / 2}(B / 10 \mu \mathrm{G})^{-3 / 2} \mathrm{~cm}$. For particles radiating their peak at $4 \mathrm{keV}$ in a magnetic field of $100 \mu \mathrm{G}$ (somewhat higher than estimates for SN 1006 based on rim widths, e.g., Parizot et al. 2006), $r_{g}=1.5 \times 10^{15} \mathrm{~cm}$, or about 0 '.05 at $2.2 \mathrm{kpc}$ - far too small to be detectable even from Chandra.

The diffusive scale length is considerably longer, however. For Bohm diffusion, the mean free path $\lambda$ is just $r_{g}$, and $\kappa=\lambda v / 3=r_{g} c / 3$, so the length scale is longer than the gyroradius by $c / 3 v_{\text {shock}}$. For $5000 \mathrm{~km} \mathrm{~s}^{-1}$ shock in $\mathrm{SN} 1006$, the diffusive scale is thus $20 r_{g}$ or about $3 \times 10^{16} \mathrm{~cm} \approx 1^{\prime \prime} \approx 2$ ACIS pixels. This is the solution favored by Morlino et al. (2010). Our data do not rule out a halo this narrow. A halo on the $10^{\prime \prime}$ scale, well above what our data allow, would require (for the same factor of $\left.c / 3 v_{\text {shock }}=20\right) r_{g} \sim 1.5 \times 10^{16} \mathrm{~cm}$ for $4 \mathrm{keV}$ electrons, and hence $B \sim 22 \mu \mathrm{G}$. Similarly, a halo on a $5^{\prime \prime}$ scale would require $B \sim 34 \mu \mathrm{G}$. Detection of a halo on these scales would clearly demonstrate the presence of substantial magneticfield amplification in the shock precursor. Profile E-2 may pose the most stringent constraints, as it appears to show no evidence of halo emission at all, within the limits of our observations. A precursor narrower than $1^{\prime \prime}$ requires a precursor magnetic field of at least $79 \mu \mathrm{G}$.

Thus, synchrotron emission at $4 \mathrm{keV}$ produced by electrons accelerated in a cosmic-ray-modified shock with strong 
nonlinear magnetic-field amplification (e.g., Bell 2004) can barely be accommodated within our observations. However, since the diffusion coefficient, and hence the halo width, is expected to rise with energy, the halo could become extended enough to observe for photon energies above $4 \mathrm{keV}$. For long enough integration times, this might be possible with Chandra.

If the halo scale is larger than about $1^{\prime \prime}$, a large jump in emissivity at the viscous subshock would be necessary to explain our observations. The particle distribution is continuous at the subshock in virtually all models, so any emissivity jump would reflect the magnetic field only. For a parallel, modified shock, magnetic-field amplification might take place gradually in the precursor, in which case no jump in emissivity would be expected at the viscous subshock. In this case, only the "too narrow" option is available to accommodate our observations. The synchrotron emissivity is $j_{v} \propto B^{1+\alpha}$, where $\alpha$ is the radio spectral index. For SN 1006, $\alpha \cong 0.55$ (Green 2009), so the maximum jump in $j_{v}$ occurs for a perpendicular shock, in which case $B$ would rise by a factor equal to the compression ratio, in the absence of additional downstream amplification processes. For an unmodified shock that factor is $4^{1.55}=8.6$, not nearly enough to explain the jumps we see. However, it is also possible that any magnetic-field amplification would take place behind the subshock (e.g., Giacalone \& Jokipii 2007), in which case an emissivity jump at the subshock could be large. If instead the shock is a modified one, the overall compression ratio is larger, but the shock transition is now broader, with the viscous subshock (with compression ratio <4) presumably accounting for the sudden emissivity jump-so the predicted step at the subshock would be even smaller, and inconsistent with our results. Independent of models, a jump by a factor of $\gtrsim 20$ in emissivity requires a jump in magnetic field by a factor of $\gtrsim 7$.

To summarize, we see minimal indication of emission beyond a sudden steep rise which we presume to indicate the viscous shock. Any upstream emission is either confined to within $\lesssim 3^{\prime \prime}$ of that rise, or fainter than about $1 \%$ of the peak. The "too narrow" option requires either a well-ordered magnetic field perpendicular to the shock velocity at all of our profile locations (disfavored by radio data; Reynoso et al. 2013), or a magnetic field that is substantially amplified in the precursor over expected ISM values. The "too faint" option requires some process greatly increasing the magnetic field at the viscous subshock, but not before. An unresolved precursor, with the magnetic field growing by (at least) a factor of seven over the far-upstream value, is the most straightforward interpretation of our data.

\section{DISCUSSION}

Previous work (e.g., Koyama et al. 1995; Dyer et al. 2001; Long et al. 2003; Yamaguchi et al. 2008) has demonstrated conclusively that almost all the thermal X-ray emission in SN 1006 is due to ejecta, giving us the opportunity to learn about the thermonuclear event that produced the remnant, and about evolutionary processes operating early in the interaction of the ejecta with the surrounding medium. The data sets we present in this paper allow us to locate the forward shock conclusively around most of the rim through the $\mathrm{H} \alpha$ emission, and to locate ejecta through the bulk of the thermal X-ray emission (though some $\mathrm{O}$ and $\mathrm{Mg}$ may also arise from an ISM component). A low density or low ionized fraction can preclude Balmer emission, but where such emission is seen its presence indicates the outermost shock, whether at the limb or in projection against the interior.

\subsection{Outer Blast Wave and ISM Interaction}

The presence of Balmer filaments, though faint, around virtually the entire limb indicates that there is at least partially neutral material all around the periphery of SN 1006, and features projected against the remnant allow us to identify material on the front or back surfaces of the remnant in contrast to material in the interior. We locate the shock in the SE, where ejecta plumes appear to reach to within $3^{\prime \prime}-30^{\prime \prime}$ of the shock. We also identify bowshocks in $\mathrm{H} \alpha$ and X-rays, the former evidently due to ejecta knots beyond the mean shock surface, as seen extending beyond the remnant edge in several locations and also in some interior locations (see Section 4 and Figure 4). Cassam-Chenaï et al. (2008) also remarked on the bowshocks protruding beyond the mean blastwave location. However, our deeper $\mathrm{H} \alpha$ image shows faint $\mathrm{H} \alpha$ emission at slightly larger radii than was evident in the image they displayed (from Winkler et al. 2003).

We can take advantage of Chandra's superior spatial resolution to directly measure the thermal emission from the shocked ISM in the SE. Both Acero et al. (2007) and Miceli et al. (2012) have reported an indirect spectroscopic detection of a shocked ISM component in addition to a shocked ejecta component, based on XMM-Newton spectroscopy. But with Chandra, we can directly separate out the two components spatially. We show in Figure 15 a region in the SE that was constructed to be outside of the fluffy ejecta structure, yet inside the extent of the faintest $\mathrm{H} \alpha$ shock as seen in the optical image. This region, covering $\sim 30^{\circ}$ of arc in length, ranges in thickness from $3^{\prime \prime}$ to $30^{\prime \prime}$, with an average thickness of $\sim 20^{\prime \prime}$.

The background-subtracted spectrum from this region shows extremely faint thermal emission, which we fit with an absorbed plane-shock model. For our purposes here, we are most interested in the emission measure $\left(\equiv n_{e} n_{p} V\right.$, where $n_{e}$ and $n_{p}$ are the electron and proton densities, respectively, and $V$ is the volume of the emitting region), which we find to be $3.7 \times 10^{54} \mathrm{~cm}^{-3}$. We estimate the line-of-sight depth through the emitting region to be half the length of the region, making $V \approx 9 \times 10^{55} \mathrm{~cm}^{3}$. Assuming cosmic abundances, where $n_{e}=1.2 n_{p}$, and a filling fraction for the gas of unity, we obtain a mean post-shock proton density of $0.18_{-0.08}^{+0.20} \mathrm{~cm}^{-3}$, temperature $k T \approx 0.80 \mathrm{keV}$, and ionization timescale $n_{e} t \approx 3.9 \times 10^{8} \mathrm{~cm}^{-3} \mathrm{~s}$. Assuming the standard compression ratio for a strong shock of 4 , this leads to a pre-shock density of $n_{0}=0.045_{-0.020}^{+0.049} \mathrm{~cm}^{-3}$, comparable with the results obtained in the XMM-Newton analyses. Cosmic-ray modification of the shock, which Miceli et al. (2012) found to be consistent with their spectral fits along the SE rim, would lower this pre-shock density, since it would raise the compression ratio of the shock.

While the value obtained above for the ISM density along the SE rim is low, it is not surprisingly so for a region far above the Galactic plane, and is consistent with previous preshock density determinations for SN 1006 (for regions other than along the NW bright filament, where considerably higher pre-shock densities have been measured, $n_{0} \approx 0.15-0.3 \mathrm{~cm}^{-3}$; Winkler et al. 2013 and numerous references therein). Long et al. (2003) estimated an upper limit of $0.1 \mathrm{~cm}^{-3}$ in the NE synchrotron-dominated limb, consistent with the value we find here for the SE. Miceli et al. (2012) fit data from deep XMMNewton observations of the SE region using a multi-component shock model with two non-equilibrium ionization components 


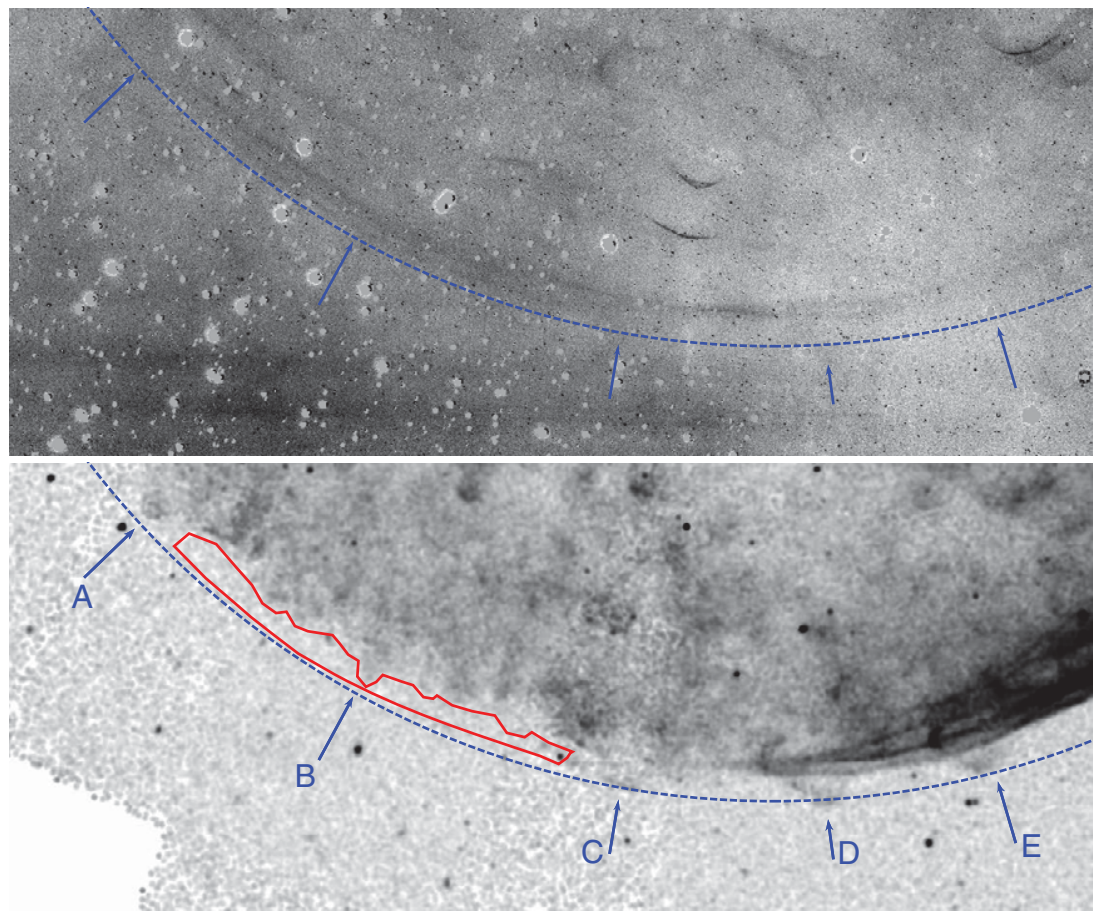

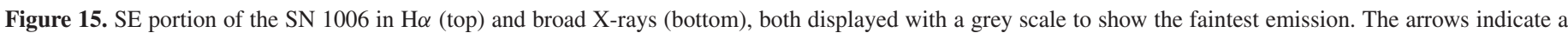

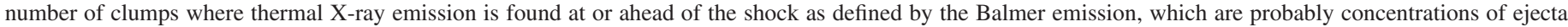

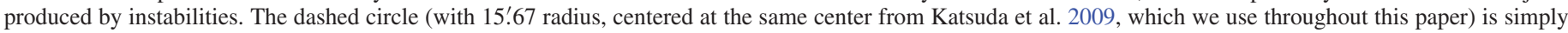

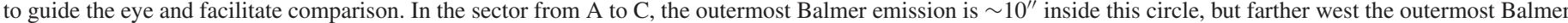

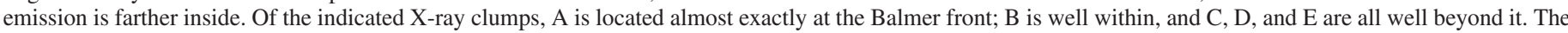

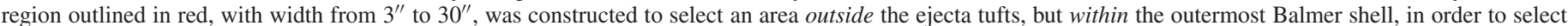
for shocked ISM, as described in the text.

(A color version of this figure is available in the online journal.)

(model vpshock in XSPEC) plus a non-thermal component, to spectroscopically separate the ejecta (with variable abundances) from the shocked ISM (abundances fixed at solar). From the solar-abundance component, they estimated a pre-shock density of $\lesssim 0.05 \mathrm{~cm}^{-3}$, consistent with our results and with an earlier XMM-Newton analysis by Acero et al. (2007). In view of the potential ambiguities that attend analyses using multiple components (including those by Long et al., Acero et al., and Miceli et al.), our analysis of emission that originates beyond the ejecta plumes should provide a cleaner limit on the ISM density.

\subsection{Large-scale Ejecta Distribution}

The images of Figure 9 show immediately that the ejecta distribution is not symmetric, with Si much brighter in the SE than in the NW, confirming the Suzaku result recently reported by Uchida et al. (2013). As in that paper, we see that $\mathrm{O}$ is distributed more uniformly than Si. Indeed, for the bright X-ray filament along the NW, we found in Winkler et al. (2013, based on a reanalysis of the archival data used by Long et al. 2003) that solar abundances can describe the X-ray emission, indicating a significant contribution from interstellar oxygen. However, Si is supersolar over most of the remnant, as expected for SN Ia ejecta; its highly asymmetric distribution indicates substantial asymmetries in the ejecta in general. While ionization effects can also cause variations in the strength of Si lines, suppressing $\mathrm{Si} \mathrm{K} \alpha$ emission in the NW as much as we observe would require a far lower ionization timescale than observed there $\left(\sim 2 \times 10^{9} \mathrm{~cm}^{-3} \mathrm{~s}\right.$; Long et al. 2003). Nonuniform distributions of emission from $\mathrm{O}, \mathrm{Ne}$, and $\mathrm{Mg}$, as well as reported distributions of Fe from Suzaku (Yamaguchi et al. 2008), all support the necessity for asymmetric ejecta.

Asymmetries, attributed to the explosion itself, are also indicated from UV absorption-line spectroscopy of UV-bright point sources behind SN1006 (e.g., Hamilton et al. 1997; Winkler et al. 2005; Hamilton et al. 2007; Winkler et al. 2011). These spectra show large differences in the column density of Fe II in the freely expanding ejecta along lines of sight separated by several arcminutes, as well as strongly asymmetric profiles of Fe II and Si II. In particular, a sharp red edge in the Si II $\lambda 1260$ absorption profile for the Schweizer \& Middleditch (1980) star indicates that the fastest unshocked ejecta (i.e., material just encountering the reverse shock) on the far side of the SNR is traveling outward at $7000 \mathrm{~km} \mathrm{~s}^{-1}$, but that such ejecta on the near side is moving far slower.

We therefore consider models for the distribution of ejecta in SN Ia explosions. Various authors (e.g., Kasen et al. 2009; Maeda et al. 2010; Seitenzahl et al. 2013) have demonstrated in recent years that two-dimensional and three-dimensional calculations of Type Ia explosions can produce somewhat different results from those of classic spherically symmetric models such as W7 of Nomoto et al. (1984)_-both in nucleosynthetic yields and in kinematic distribution. The three-dimensional delayeddetonation models of Seitenzahl et al. (2013) predict a range of $\mathrm{O}$ and $\mathrm{Si}$ ratios and locations. After about $100 \mathrm{~s}$, ejecta are in ballistic motion (i.e., pressure forces are negligible and material is freely expanding). At that time, for the most asymmetric models (ones with the fewest ignition points), azimuthally averaged $\mathrm{O}$ and intermediate-mass elements have similar distributions, while for more symmetric models, $\mathrm{O}$ is found at considerably larger distances than $\mathrm{Si}$. The strong $\mathrm{Si}$ asymmetry we find is 
most easily explained by their very asymmetric model N3 (see their Figure 3); we would then interpret our more uniform $\mathrm{O}$ distribution as an indication of substantial shocked-ISM oxygen. $\mathrm{Mg}$ is intermediate; the models of Iwamoto et al. (1999) produce three to eight times as much $\mathrm{Mg}$ as $\mathrm{Ne}$, so we might expect to find a substantial component of $\mathrm{Mg}$ from ejecta in addition to some that has resulted from shocked ISM. All this is consistent with the visual impression from Figure 9. We conclude that the ejecta distribution in SN 1006 qualitatively supports quite asymmetric SN Ia models, though detailed quantitative spectral analysis and modeling will be required to make this statement more definite.

\subsection{Small-scale Ejecta Distribution}

The arcminute-scale "puffy" structure of ejecta apparent in SN 1006 X-ray images has been noted by many authors. Its origin could be in intrinsic clumpiness produced in the explosion itself ("intrinsic" clumps) or in hydrodynamically produced structures from R-T instabilities at the contact interface (R-T clumps). Intrinsic clumps could arise if the ejecta are subject to the "nickel bubble" effect (Li et al. 1993) in which $\gamma$-rays from the radioactive decay of ${ }^{56} \mathrm{Ni}$ cause local expansion of the ejecta and sweep surrounding ejecta into a shell, which is then fragmented by R-T instabilities in the first few minutes after the explosion. Later developing R-T clumps are seen in all hydrodynamic simulations of SNR evolution (e.g., Chevalier et al. 1992; Jun \& Norman 1996; Orlando et al. 2012; Warren \& Blondin 2013). Determining which process produces the clumpy structure in SN 1006 is important, as intrinsic clumps can be used as diagnostics of the explosion, while R-T clumps contain information on the global hydrodynamic evolution. Unfortunately, the two models typically produce structures of similar appearance: R-T clumps are formed from dense "mushroom caps" of less decelerated material, and thus appear disk-like, while intrinsic clumps are flattened to a similar shape as they are slowed and eventually fragmented by instabilities (Wang \& Chevalier 2001; Orlando et al. 2012). Such morphologies would result in structures much more easily visible from the side (due to longer lines of sight through material) than from face on, and can explain the lack of small-scale $\mathrm{H} \alpha$ emission toward the center of SN 1006.

Our observations of numerous bowshock structures in $\mathrm{H} \alpha$ (Section 4) illustrate that it is relatively common for ejecta clumps to reach the outer blast wave and interact with at least partially neutral material. Such structures are often described as ejecta "bullets" or "shrapnel" (e.g., Wang \& Chevalier 2001; Miceli et al. 2013b). Those seen in projection against the remnant interior (see Figure 4) present serious challenges to $\mathrm{X}$-ray spectral analysis due to projection effects. Particularly useful are the structures that can be seen at the extreme edge of the remnant, where they clearly extend beyond the mean blast-wave radius.

The very existence of such protrusions poses significant difficulties for models. The overall puffy structure of ejecta in SN 1006 strongly resembles the simulated images from threedimensional hydrodynamic simulations by Warren \& Blondin (2013). Those simulations evolved an initial exponential density profile into a uniform ambient medium; the ejecta structure was produced purely by hydrodynamic instabilities. But the simulations of Warren \& Blondin (2013) are able to produce clumps breaking through the mean shock radius only if the shock compression is quite high (simulated by an artificially low adiabatic index; $\gamma=6 / 5$ giving a compression ratio of 11), explained by efficient cosmic-ray acceleration. A high compression ratio provides a less likely explanation in the SE, where the relativistic-particle population is evidently less important, judging by the relative weakness of nonthermal emission at radio, X-ray, and gamma-ray wavelengths.

Intrinsic clumpiness may also be able to produce similar projections, however. If such clumps are sufficiently dense (Wang \& Chevalier 2001, find a density contrast of $\sim 100$ is required for structures seen in Tycho's SNR), they would be less decelerated and capable of producing the structures we see in SN 1006. In the MHD simulations of Orlando et al. (2012), magnetic-field amplification at the outer edges of clumps stabilizes these structures and enables some clumps with a density contrast of a factor of only $\lesssim 10$ to reach or surpass the mean blast-wave radius, without resorting to unusually compressive shocks. The connection between the close approach of the contact discontinuity to the forward shock and efficient shock acceleration, as was favored by Cassam-Chenaï et al. (2008), is no longer required. Detailed spectral analysis of features beyond the blast wave, and of sufficiently bright $\mathrm{X}$-ray structures behind bowshocks seen against the interior, may allow a discrimination between the intrinsic versus latertime R-T models.

\section{SUMMARY AND CONCLUSIONS}

Here we have provided an overview of new X-ray and optical observations of the remnant of SN 1006. X-ray emission in SN 1006 is complex: the interior of the SNR is filled with small-scale features arising primarily from SN ejecta, while the shell rims exhibit both synchrotron radiation from electrons accelerated at the shock front (in the NE and SW), and thermal X-ray emission from hot plasma-both SN ejecta and shocked ISM-in the NW and SE. Our primary results are as follows.

1. $\mathrm{H} \alpha$ emission can be traced around almost the entire SNR shell, even in regions that are dominated by synchrotron radiation. Very faint, diffuse $\mathrm{H} \alpha$ emission, arising from the near and/or far side of the SNR, covers a substantial portion of the interior of the SNR. Wherever it is found, the short lifetime for neutral $\mathrm{H}$ atoms behind fast shocks like those in SN 1006 requires that the Balmer-line emission must occur immediately behind a shock encountering partially neutral ISM.

2. Some of the small-scale $\left(10^{\prime \prime}-30^{\prime \prime}=0.1-0.3 \mathrm{pc}\right) \mathrm{X}$-ray features within the (projected) SNR shell have associated Balmer filaments that resemble bow shocks. The X-ray spectra of these tuft-like features shows that they are ejecta and that they have ionization timescales that are short compared to the time since the SN exploded. The fact that these features have associated Balmer emission indicates that they are ejecta "bullets" that are penetrating the interstellar shock and encountering pristine ISM. Further analysis will be required to determine whether these ejecta knots are a result of density inhomogeneities originating in the SN explosion, or have been produced later through R-T instabilities as the SNR has evolved.

3. The expansion velocity of the outer edge of the SNR, as measured from the proper motion, varies dramatically as a function of azimuth. The lowest velocity is about $3000 \mathrm{~km} \mathrm{~s}^{-1}$ in the $\mathrm{NW}$, where the strongest $\mathrm{H} \alpha$ emission occurs, and where the pre-shock density is highest. In the $\mathrm{SE}$, the velocity is almost 2.5 times higher, $\sim 7400 \mathrm{~km} \mathrm{~s}^{-1}$. The synchrotron-dominated limbs in the NE and SW both have velocities of about $5000 \mathrm{~km} \mathrm{~s}^{-1}$. 
4. The overall distribution of ejecta material is asymmetric. We confirm the results of Uchida et al. (2013) that silicon, which arises almost entirely from SN ejecta, is strongly concentrated in the SE quadrant. Emission from neon is by far the strongest along the NW rim, where the primary shock is encountering denser material than anywhere else around the periphery. Oxygen and magnesium show less extreme concentration in the SE than does silicon, and also show concentrations well inside the shell rim in the NW quadrant; these probably arise from a mix of SN ejecta and shocked ISM.

5. Our data place significant constraints on a possible X-ray halo in front of any of the synchrotron-dominated regions along the NE or SW limbs. We observe abrupt jumps in emission by a factor ranging from $\sim 20$ to $>100$ over scales comparable with the PSF for the instrument at multiple locations. Immediately preceding these jumps, there is slight evidence for a faint precursor on scales of $\lesssim 3^{\prime \prime}$. The most straightforward explanation of these results is that diffusive particle acceleration is promoted by a magnetic field that is amplified by a factor of seven or more in a narrow precursor region.

6. Even in the new, deep Chandra images, there is no clear evidence for the primary shock along the rim of the SNR shell in the SE. Instead, the X-ray structure there consists of a series of tufts, whose kinematics suggest that they have been decelerated little if at all (expansion index $m \approx 0.8$ ) and whose spectra show they are ejecta-dominated. Within a narrow region ahead of the X-ray tufts, but behind the outermost $\mathrm{H} \alpha$ emission, we find extremely faint thermal $\mathrm{X}$-ray emission whose emission measure suggests a preshock density $n_{0} \approx 0.045 \mathrm{~cm}^{-3}$, similar to the value inferred by other investigators through different arguments.

The data set from the Chandra Large Project to survey SN 1006 is a rich one, and should provide a resource for many future studies, by ourselves and others.

We acknowledge several suggestions from the anonymous referee that have led to greater clarity in this paper. Support for this work was provided by the National Aeronautics and Space Administration through Chandra Grant Number GO2-13066, issued by the Chandra X-ray Observatory Center, which is operated by the Smithsonian Astrophysical Observatory for and on behalf of NASA under contract NAS8-03060. P.F.W. also acknowledges financial support from the National Science Foundation through grant AST-0908566.

\section{REFERENCES}

Acero, F., Ballet, J., \& Decourchelle, A. 2007, A\&A, 475, 883

Bamba, A., Fukazawa, Y., Hiraga, J. S., et al. 2008, PASJ, 60, 153

Becker, R. H., Szymkowiak, A. E., Boldt, E. A., Holt, S. S., \& Serlemitsos, P. J. 1980, ApJL, 240, L33

Bell, A. R. 2004, MNRAS, 353, 550

Broersen, S., Vink, J., Miceli, M., et al. 2013, A\&A, 552, A9

Cassam-Chenaï, G., Hughes, J. P., Reynoso, E. M., Badenes, C., \& Moffett, D. 2008, ApJ, 680, 1180

Chevalier, R. A., Blondin, J. M., \& Emmering, R. T. 1992, ApJ, 392, 118

Chevalier, R. A., \& Raymond, J. C. 1978, ApJL, 225, L27

Dubner, G. M., Giacani, E. B., Goss, W. M., Green, A. J., \& Nyman, L.-A. 2002, A\&A, 387, 1047
Dyer, K. K., Reynolds, S. P., Borkowski, K. J., Allen, G. E., \& Petre, R. 2001, ApJ, 551, 439

Ellison, D. C., Baring, M. G., \& Jones, F. C. 1996, ApJ, 473, 1029

Forman, W., Jones, C., Cominsky, L., et al. 1978, ApJS, 38, 357

Ghavamian, P., Winkler, P. F., Raymond, J. C., \& Long, K. S. 2002, ApJ, 572,888

Giacalone, J., \& Jokipii, J. R. 2007, ApJL, 663, L41

Green, D. A. 2009, BASI, 37, 45

Hamilton, A. J. S., Fesen, R. A., \& Blair, W. P. 2007, MNRAS, 381, 771

Hamilton, A. J. S., Fesen, R. A., Wu, C.-C., Crenshaw, D. M., \& Sarazin, C. L. 1997, ApJ, 481, 838

Heng, K. 2010, PASA, 27, 23

Hester, J. J. 1987, ApJ, 314, 187

Hwang, U., Holt, S. S., \& Petre, R. 2000, ApJL, 537, L119

Iwamoto, K., Brachwitz, F., Nomoto, K., et al. 1999, ApJS, 125, 439

Jun, B.-I., \& Norman, M. L. 1996, ApJ, 465, 800

Kasen, D., Röpke, F. K., \& Woosley, S. E. 2009, Natur, 460, 869

Katsuda, S., Long, K. S., Petre, R., et al. 2013, ApJ, 763, 85

Katsuda, S., Petre, R., Long, K. S., et al. 2009, ApJL, 692, L105

Katsuda, S., Petre, R., Mori, K., et al. 2010, ApJ, 723, 383

Koyama, K., Petre, R., Gotthelf, E. V., et al. 1995, Natur, 378, 255

Li, H., McCray, R., \& Sunyaev, R. A. 1993, ApJ, 419, 824

Long, K. S., Blair, W. P., \& van den Bergh, S. 1988, ApJ, 333, 749

Long, K. S., Reynolds, S. P., Raymond, J. C., et al. 2003, ApJ, 586, 1162

Maeda, K., Röpke, F. K., Fink, M., et al. 2010, ApJ, 712, 624

Miceli, M., Bocchino, F., Decourchelle, A., et al. 2012, A\&A, 546, A66

Miceli, M., Bocchino, F., Decourchelle, A., et al. 2013a, A\&A, 556, A80

Miceli, M., Orlando, S., Reale, F., Bocchino, F., \& Peres, G. 2013b, MNRAS, 430, 2864

Morlino, G., Amato, E., Blasi, P., \& Caprioli, D. 2010, MNRAS, 405, L21

Nikolić, S., van de Ven, G., Heng, K., et al. 2013, Sci, 340, 45

Nomoto, K., Thielemann, F.-K., \& Yokoi, K. 1984, ApJ, 286, 644

Orlando, S., Bocchino, F., Miceli, M., Petruk, O., \& Pumo, M. L. 2012, ApJ, 749, 156

Palmieri, T. M., Burginyon, G. A., Hill, R. W., Scudder, J. K., \& Seward, F. D. 1972, ApJ, 177, 387

Parizot, E., Marcowith, A., Ballet, J., \& Gallant, Y. A. 2006, A\&A, 453, 387

Pye, J. P., Pounds, K. A., Rolf, D. P., et al. 1981, MNRAS, 194, 569

Raymond, J. C., Korreck, K. E., Sedlacek, Q. C., et al. 2007, ApJ, 659,1257

Reynolds, S. P. 1996, ApJL, 459, L13

Reynoso, E. M., Hughes, J. P., \& Moffett, D. A. 2013, AJ, 145, 104

Schweizer, F., \& Lasker, B. M. 1978, ApJ, 226, 167

Schweizer, F., \& Middleditch, J. 1980, ApJ, 241, 1039

Seitenzahl, I. R., Ciaraldi-Schoolmann, F., Röpke, F. K., et al. 2013, MNRAS, 429, 1156

Stephenson, F. R., \& Clark, D. H. 2002, Historical Supernovae and Their Remnants (Oxford: Oxford Univ. Press), 150

Uchida, H., Yamaguchi, H., \& Koyama, K. 2013, ApJ, 771, 56

van Adelsberg, M., Heng, K., McCray, R., \& Raymond, J. C. 2008, ApJ, 689, 1089

van den Bergh, S. 1976, ApJL, 208, L17

Vink, J., Laming, J. M., Gu, M. F., Rasmussen, A., \& Kaastra, J. S. 2003, ApJL, 587, L31

Wang, C.-Y., \& Chevalier, R. A. 2001, ApJ, 549, 1119

Warren, D. C., \& Blondin, J. M. 2013, MNRAS, 429, 3099

Willingale, R., West, R. G., Pye, J. P., \& Stewart, G. C. 1996, MNRAS, 278, 749

Winkler, P. F., Gupta, G., \& Long, K. S. 2003, ApJ, 585, 324

Winkler, P. F., Hamilton, A. J. S., Long, K. S., \& Fesen, R. A. 2011, ApJ, 742, 80

Winkler, P. F., \& Long, K. S. 1997, ApJ, 491, 829

Winkler, P. F., Long, K. S., Hamilton, A. J. S., \& Fesen, R. A. 2005, ApJ, 624, 189

Winkler, P. F., Williams, B. J., Blair, W. P., et al. 2013, ApJ, 764, 156

Winkler, P. F., Jr., Hearn, D. R., Richardson, J. A., \& Behnken, J. M. 1979, ApJL, 229, L123

Winkler, P. F., Jr., \& Laird, F. N. 1976, ApJL, 204, L111

Yamaguchi, H., Koyama, K., Katsuda, S., et al. 2008, PASJ, 60, 141

Zacharias, N., Finch, C. T., Girard, T. M., et al. 2013, AJ, 145, 44

Zacharias, N., Monet, D. G., Levine, S. E., et al. 2005, yCat, 1297, 0 